Research Article

\title{
Identification of KLF6/PSGs and NPY-Related USF2/CEACAM Transcriptional Regulatory Networks via Spinal Cord Bulk and Single-Cell RNA-Seq Analysis
}

\author{
Xinbing Liu $(\mathbb{D}$, Wei Gao $\mathbb{D}$, and Wei Liu $\mathbb{C}$ \\ Department of Neurosurgery, Changxing People's Hospital, Changxing, Zhejiang Province, China \\ Correspondence should be addressed to Wei Liu; wl@mail.alumni.edu.vn
}

Received 18 August 2021; Revised 22 October 2021; Accepted 26 October 2021; Published 29 November 2021

Academic Editor: Yuzhen Xu

Copyright (C) 2021 Xinbing Liu et al. This is an open access article distributed under the Creative Commons Attribution License, which permits unrestricted use, distribution, and reproduction in any medium, provided the original work is properly cited.

\begin{abstract}
Background. To further understand the development of the spinal cord, an exploration of the patterns and transcriptional features of spinal cord development in newborn mice at the cellular transcriptome level was carried out. Methods. The mouse single-cell sequencing (scRNA-seq) dataset was downloaded from the GSE108788 dataset. Single-cell RNA-Seq (scRNA-Seq) was conducted on cervical and lumbar spinal V2a interneurons from 2 P0 neonates. Single-cell analysis using the Seurat package was completed, and marker mRNAs were identified for each cluster. Then, pseudotemporal analysis was used to analyze the transcription changes of marker mRNAs in different clusters over time. Finally, the functions of these marker mRNAs were assessed by enrichment analysis and protein-protein interaction (PPI) networks. A transcriptional regulatory network was then constructed using the TRRUST dataset. Results. A total of 949 cells were screened. Single-cell analysis was conducted based on marker mRNAs of each cluster, which revealed the heterogeneity of neonatal mouse spinal cord neuronal cells. Functional analysis of pseudotemporal trajectory-related marker mRNAs suggested that pregnancy-specific glycoproteins (PSGs) and carcinoembryonic antigen cell adhesion molecules (CEACAMs) were the core mRNAs in cluster 3. GSVA analysis then demonstrated that the different clusters had differences in pathway activity. By constructing a transcriptional regulatory network, USF2 was identified to be a transcriptional regulator of CEACAM1 and CEACAM5, while KLF6 was identified to be a transcriptional regulator of PSG3 and PSG5. This conclusion was then validated using the Genotype-Tissue Expression (GTEx) spinal cord transcriptome dataset. Conclusions. This study completed an integrated analysis of a singlecell dataset with the utilization of marker mRNAs. USF2/CEACAM1\&5 and KLF6/PSG3\&5 transcriptional regulatory networks were identified by spinal cord single-cell analysis.
\end{abstract}

\section{Introduction}

The nervous system is composed of the central nervous system (CNS) and the peripheral nervous system (PNS). Included in the central nervous system is the spinal cord, which plays a vital role in our perception of the environment and its interactions. The spinal cord is composed of gray matter and white matter. In the center of the spinal cord, there is a thin lumen called the central canal surrounded by the ventricular epithelium. The ventricular epithelium is then surrounded by an $\mathrm{H}$-shaped gray matter followed by the white matter. The gray matter is composed of two parts, the anterior horn and the posterior horn. The anterior part of the gray matter is the anterior horn, which is larger compared to the posterior horn. There are numerous multipolar motor neurons within the anterior horn, while neurons within the posterior horn form synaptic connections with sensory ganglion cells. These neurons form a neural circuit that utilizes sensory input and converts the commands from the brain into the body's response to the environment, such as muscle contractions [1].

The discovery of the developmental trajectory of the central nervous system facilitated the exploration of the pathological process of neuronal repair; the pathogenesis of central nervous system injures and neurodegenerative diseases set the foundation for the translation of clinical 


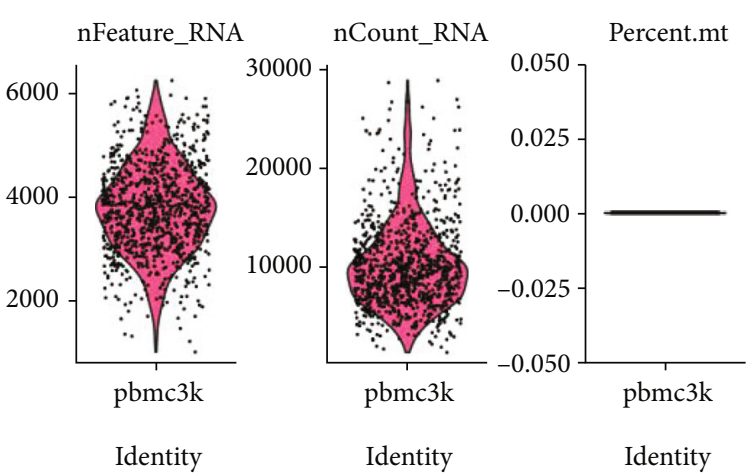

(a)

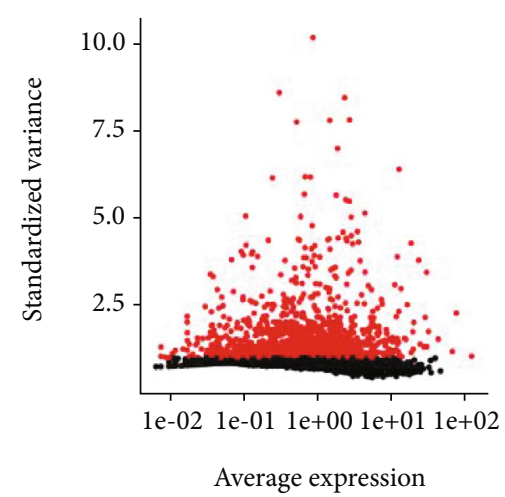

- Non-variable count: 8527

- Variable count: 2000

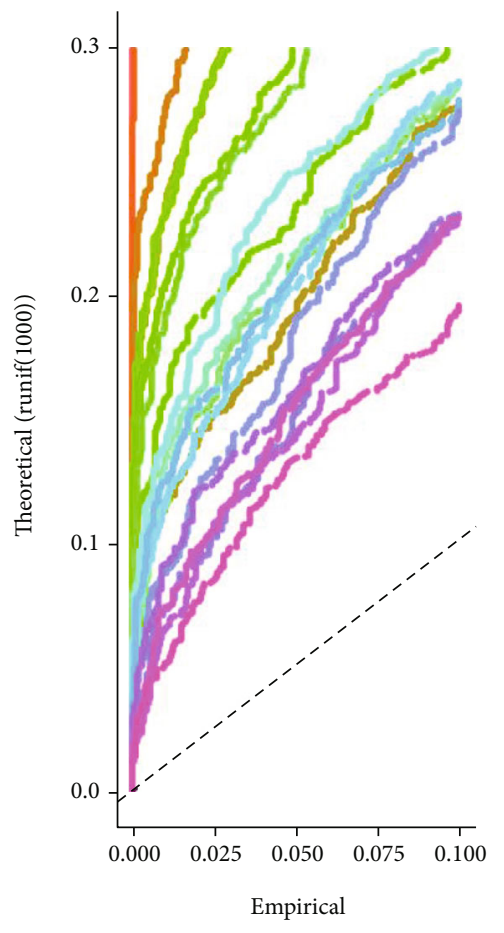

PC: p-value

- PC 1: 1.34e-27

- PC $2: 3.75 \mathrm{e}-138$

- PC 3: 1.74e-47

- PC 4: 5.75e-23

- PC 5: $2.74 \mathrm{e}-40$

- PC 6: 3.26e-63

- PC 7: $2.8 \mathrm{e}-46$

- PC 8: $4.74 \mathrm{e}-40$

- PC 19: 4.24e-39

- PC 10: 4.05e-24

- PC 11: 4.68e-28

- PC 12: 5.5e-19

- PC 13: 2.7e-19

- PC 14: 3.07e-17

- PC 15: 6.45e-13

- PC 16:3.83e-13

- PC 17: 5.8e-15

- PC 18: 1.8e-07

- PC 19: 1.63e-09

- PC 20: $1.47 \mathrm{e}-06$

(c)

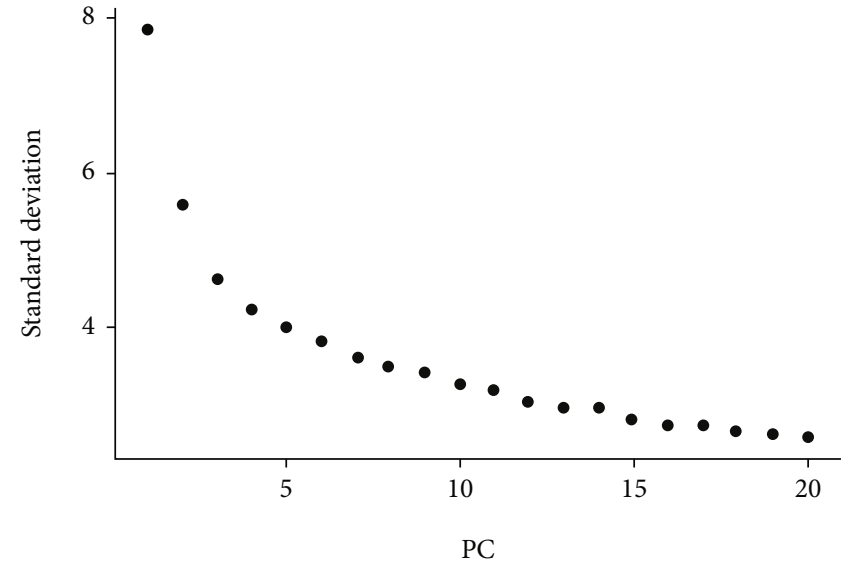

PC

(d)

Figure 1: Continued. 


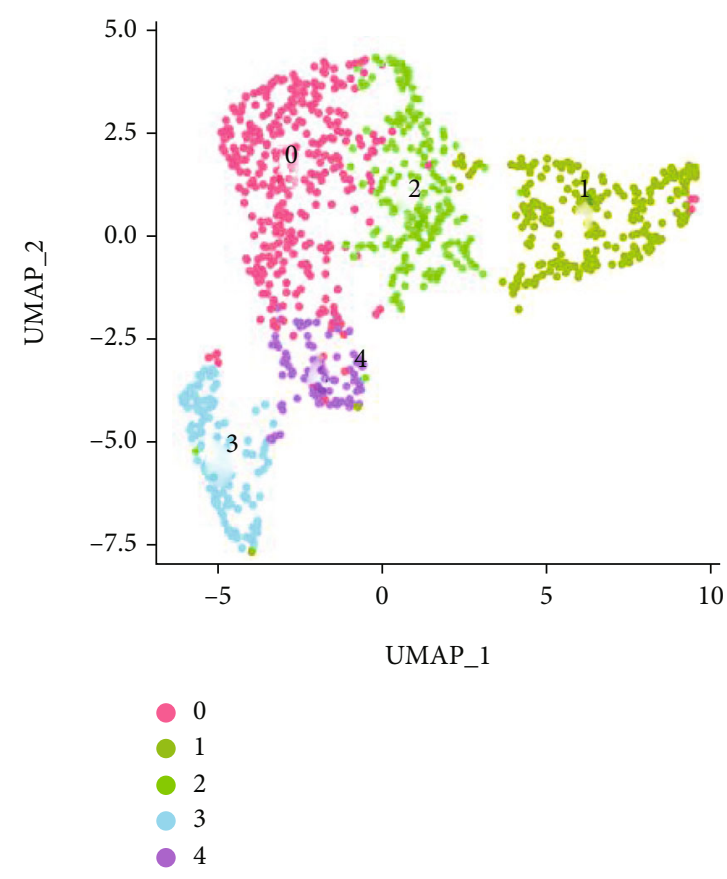

(e)
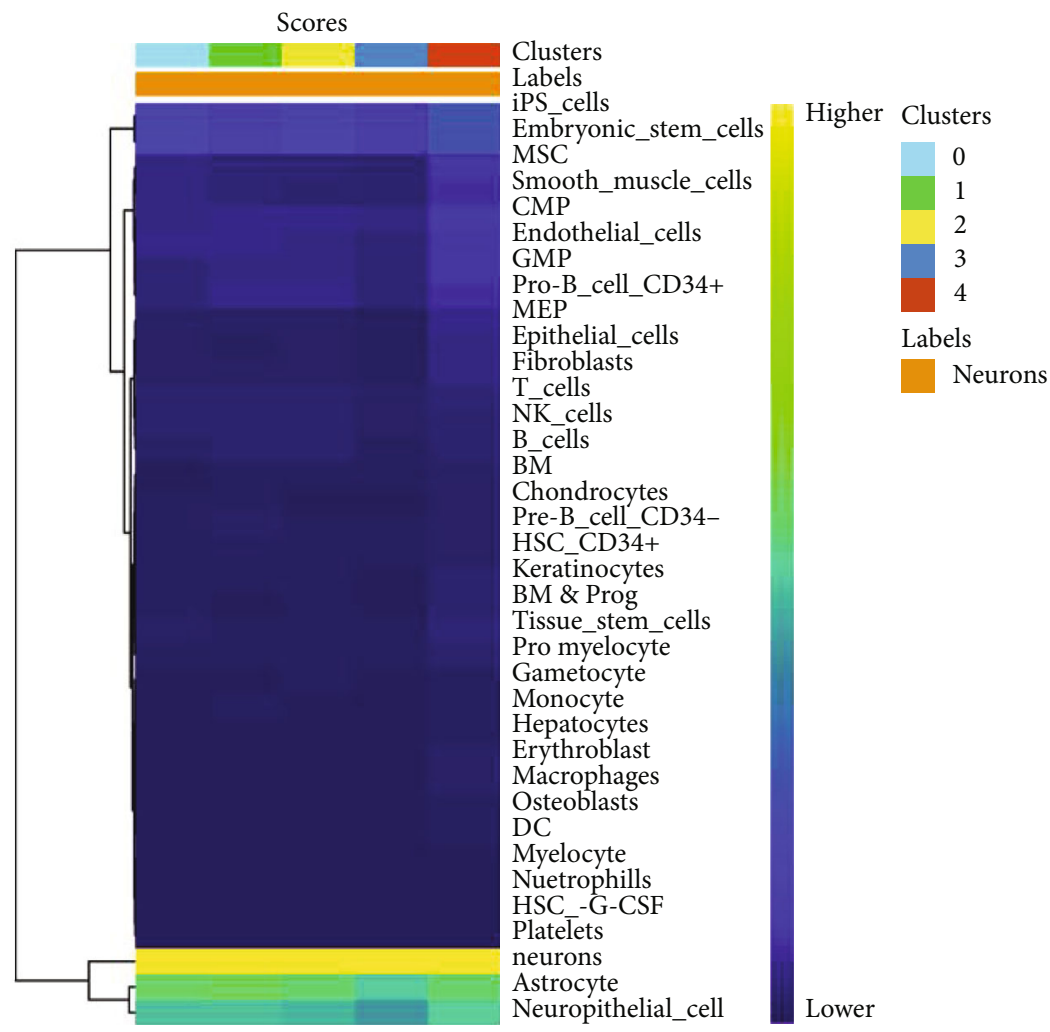

(f)

FIgURE 1: Quality control and clustering of the GSE108788 dataset. (a) Quality control process for the GSE108788 dataset. (b) ANOVA plot showing 2000 highly variable genes in mouse neuronal cells. Red indicates highly variable mRNAs; black indicates nonvariable mRNAs. (c, d) PCA analysis identified 20 clusters with $p$ values less than 0.001 . (e) The UMAP diagram shows the distribution of nerve cells into five clusters. (f) The singleR package was used to annotate the clusters, confirming that all of them belonged to neural cells.

treatment $[1,2]$. In the early stages of mouse embryonic development, the central nervous system first develops in the ectoderm. Thereafter, stem cells differentiate into a vari- ety of different cell types. However, the exact processes and steps involved in the differentiation of these cells are still controversial and have yet to be further investigated. These 


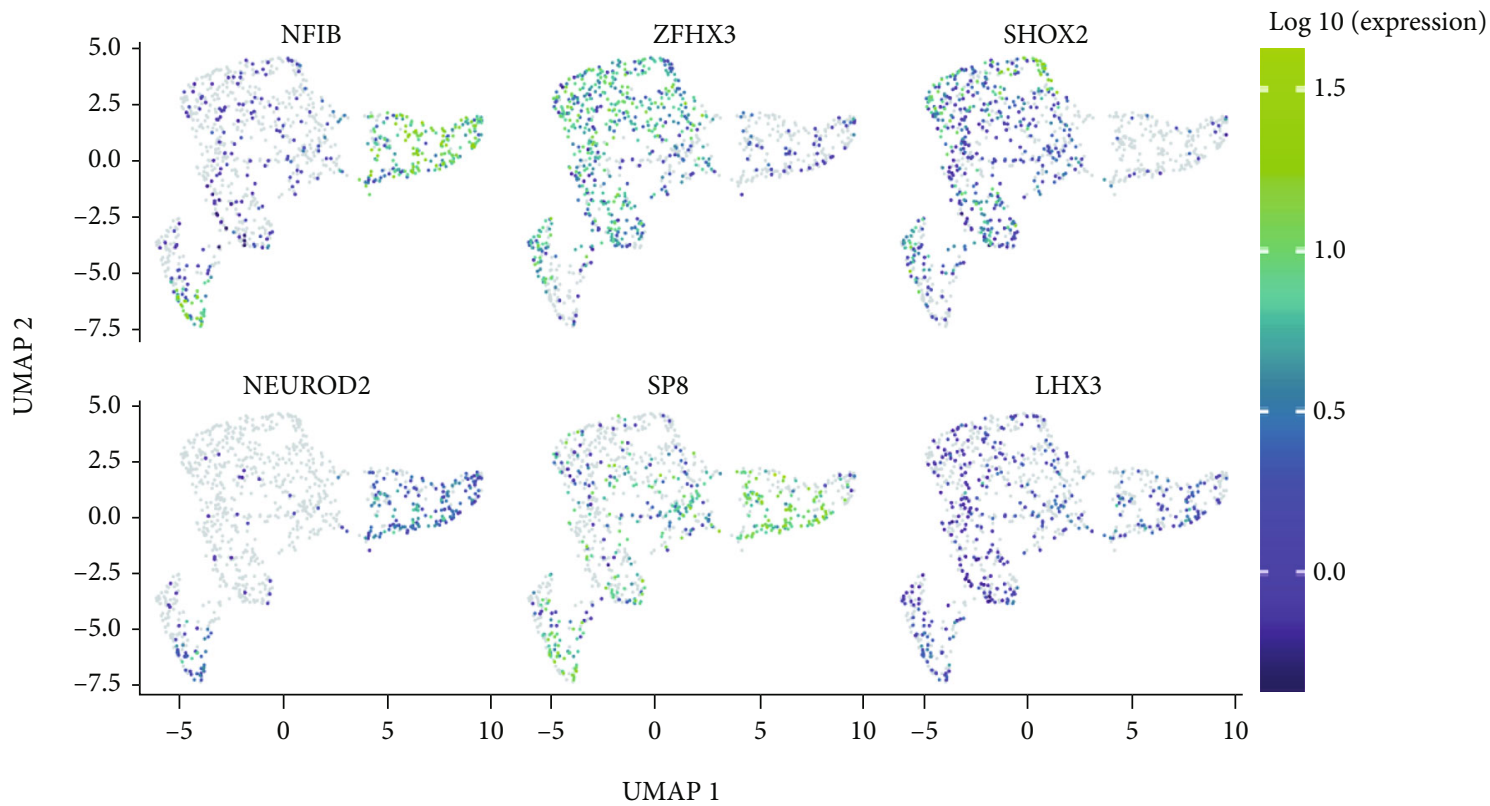

(a)

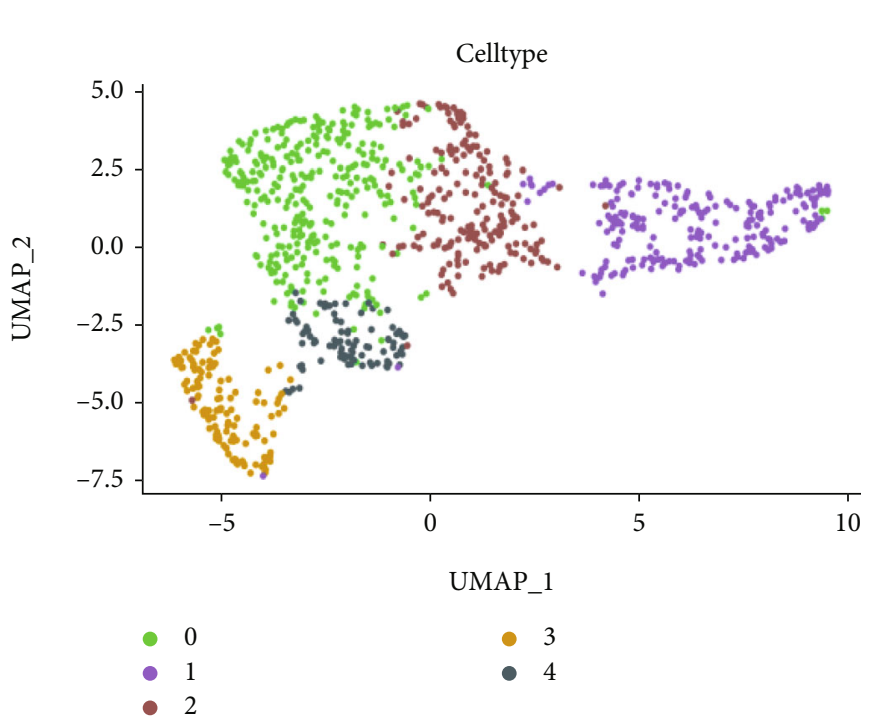

(b)

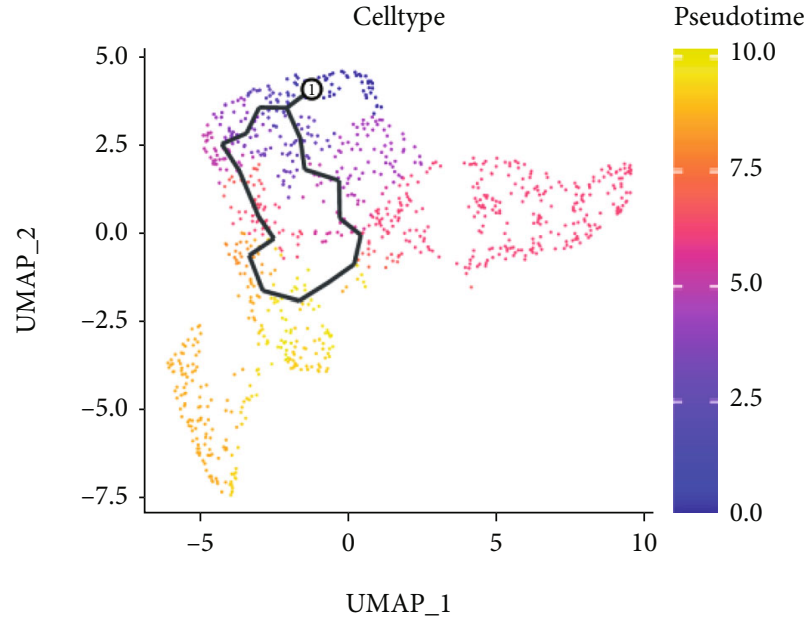

(c)

FIgure 2: Continued. 


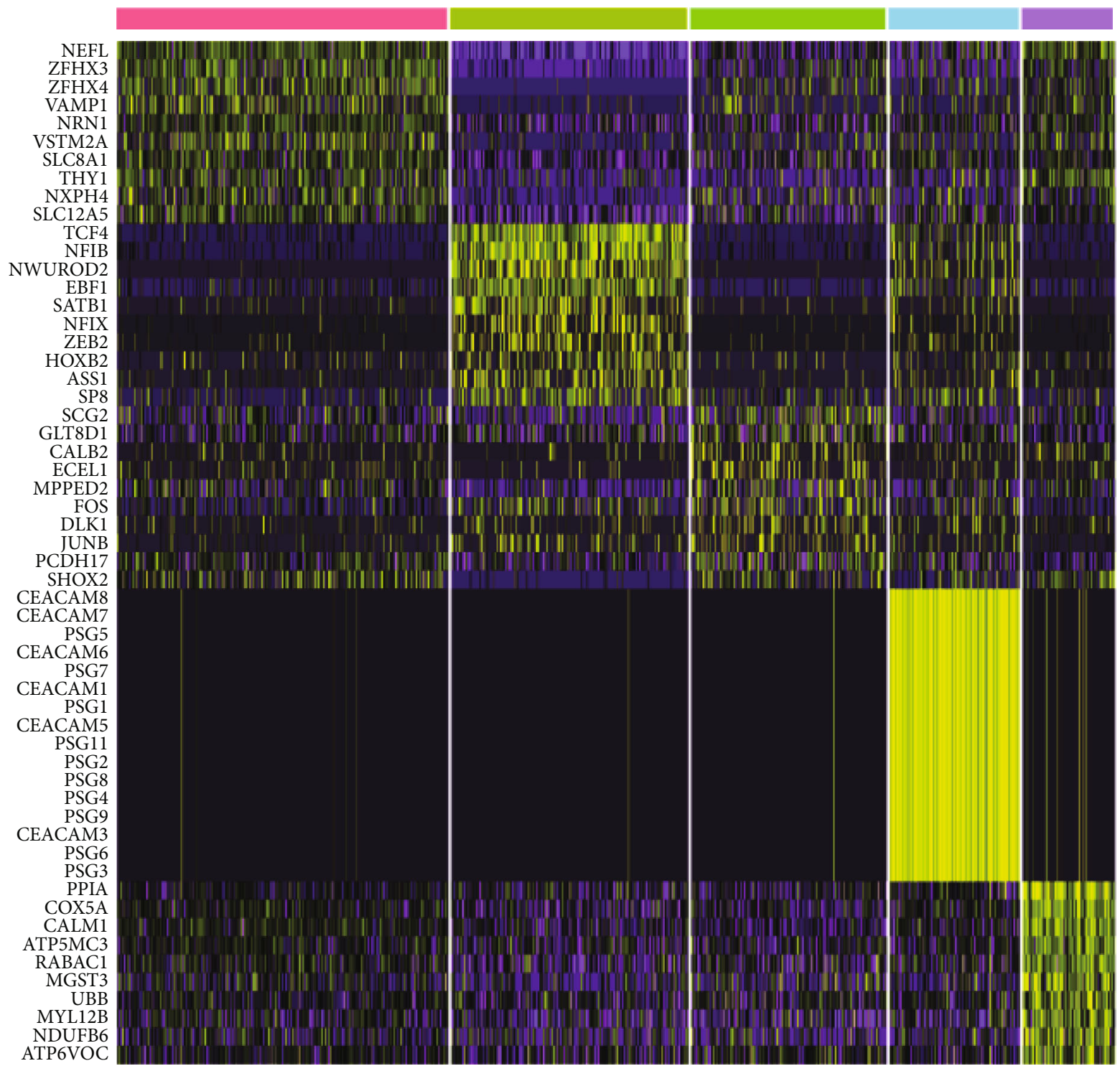

(d)

FIGURE 2: Single-cell analysis was used to reveal the heterogeneity of neonatal mouse spinal cord neuronal cells. (a) UMAP plots were used to demonstrate the expression of the markers NFIB, ZFHX3, SHOX2, NEUROD2, SP3, and LHX3. (b) These cells were divided into five cell types by PCA and UMAP analysis. (c) The top ten marker mRNAs for each cell population are shown in the heat map. (d) The proposed chronological analysis of spinal cord neuronal cells from these newborn mice.

controversies revolve around whether neural development is induced or transformed $[3,4]$. Recent studies now include the single-cell transcriptome that provides a way to precisely map the transcriptional heterogeneity of cells across time [5, 6]. As a result, CNS research has made tremendous progress in recent years due to the rapid advances in single-cell histology techniques [7].

This study is aimed at exploring the patterns and transcriptional features of spinal cord development in newborn mice at the single-cell transcriptome level, thereby furthering our current understanding of the developmental processes of the spinal cord and its underlying mechanisms. This understanding will allow the further investigation of the developmental trajectory of mouse neurons and the cell biology of this disease.

\section{Materials and Methods}

2.1. Data Collection and Computational Analysis of scRNASeq Datasets. The mouse scRNA-seq dataset was downloaded from the GEO database (GSE108788) [8]. The data from the scRNA-seq dataset was collected from neonatal mouse cervical and lumbar spine interneurons, which were sorted via flow cytometry. Quality control was carried out as described previously [8]. Similar to previous studies, the single-cell analysis was completed using the Seurat package 


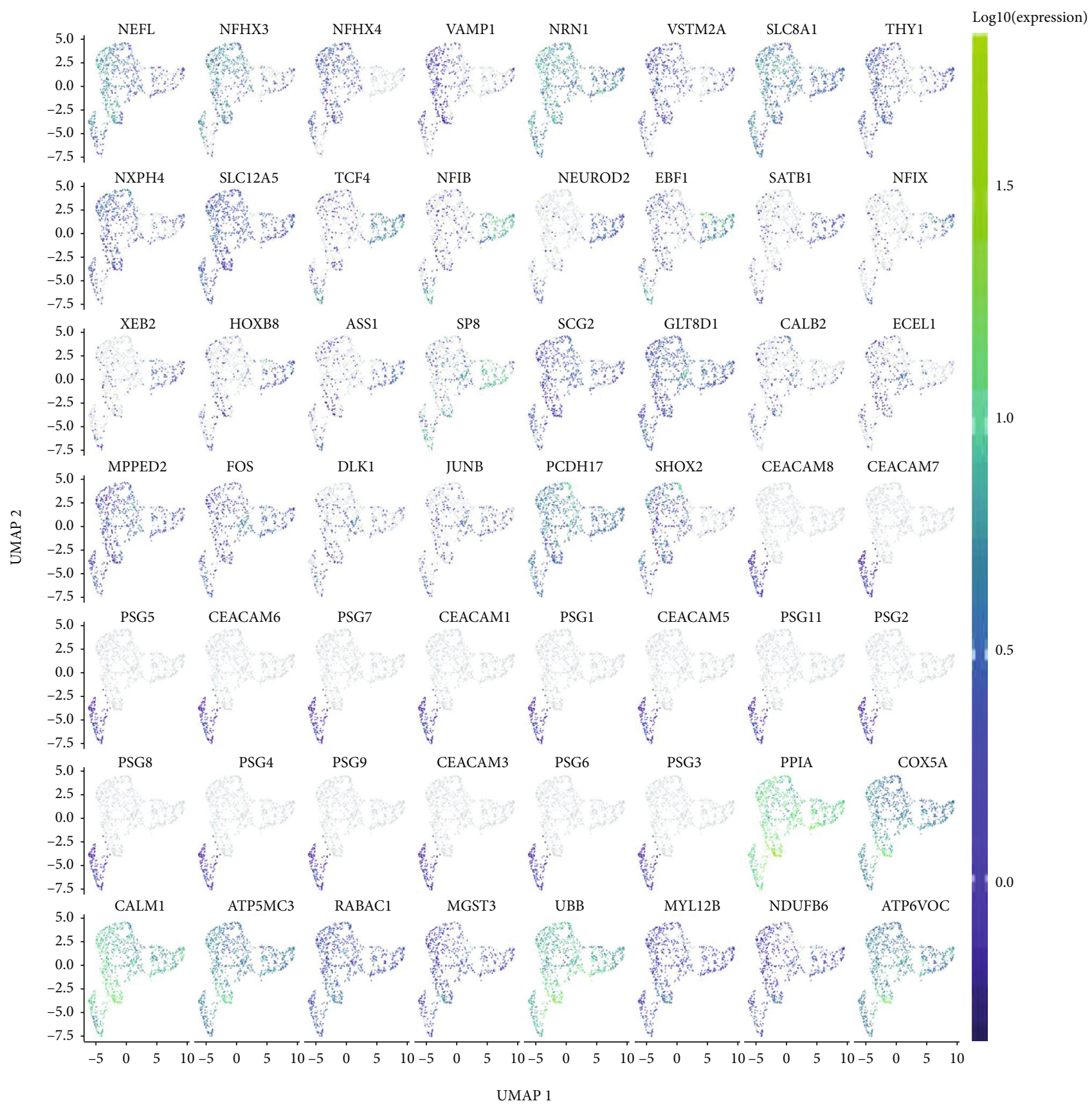

Figure 3: The UMAP plot of the markers in neonatal mouse spinal cord neuronal cells.

$[5,9]$. Briefly, the "Find neighbors" and "Find Clusters" functions from $\mathrm{R}$ software were utilized to perform principal component analysis and cell clustering [10]. The marker mRNAs between each cluster were identified using the FindAllMarkers function. Then, based on these marker mRNAs, the UMAP method was used to perform nonlinear dimensionality reduction. Initial annotation of the cells was done using the singleR package [11]. A pseudotemporal analysis was then performed using the Monocle 3 package, and the differences in expression of marker mRNAs on pseudotemporal trajectories were obtained for each cluster.
2.2. Protein-Protein Interaction (PPI) Network Analysis. The initial analysis of the PPI network was done by the STRING v11 database (http://string-db.org) (feasibility of 0.40 , medium confidence level). Then, based on the obtained results, the PPI network was constructed using the R software [12].

2.3. Enrichment Analysis. As described in the previous study, the clusterProfiler package of the R software was utilized to complete the GO and KEGG enrichment analysis of the marker mRNAs in each cluster $[13,14]$. Pathway enrichment analysis was performed with ReactomePA to identify 


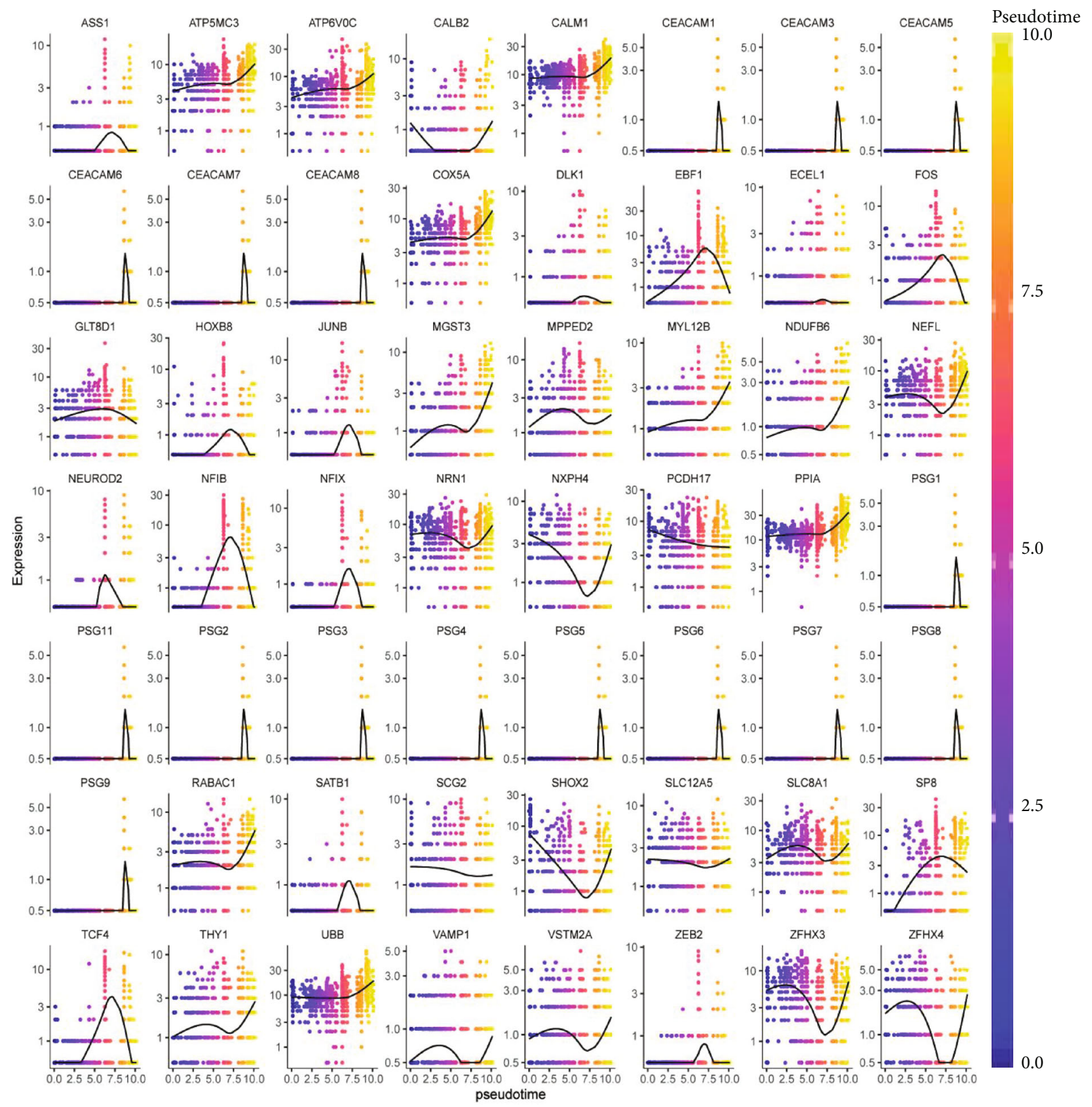

FIgURE 4: Expression of marker mRNAs varies with pseudotiming.

gene sets from the Reactome database with a false discovery rate of $<0.05$ in the marker sets $[15,16]$.

\subsection{Prediction of Transcription Factors. The TRRUST data-} base, which contains data on the regulatory relationship between 800 human transcription factors and 828 mouse transcription factors, was used for transcription factor prediction analysis. All TF-target gene pairs included were experimentally validated [17].

2.5. Statistical Analysis. Data analysis and plotting of the results were done using $\mathrm{R}$ software (version 4.0.2). The results of the intersection analysis were visualized through a Venn diagram. An unpaired $t$-test was conducted to distin- guish differences between two groups, and a $p$ value of $<0.05$ was defined as a significant difference.

\section{Results}

3.1. Quality Control and Cluster Analysis of the GSE108788 Dataset. To analyze the transcriptional characteristics of neonatal mouse neuronal cells at a single-cell level, we filtered the dataset GSE108788 and implemented quality control using the Seurat package for R software (Figure 1(a)). The selected 949 mouse neuronal cells were normalized using the Seurat package. Then, an analysis of variance (ANOVA) plot showed 2000 highly variable mRNAs (Figure 1(b)). PCA analysis was first used to screen 20 


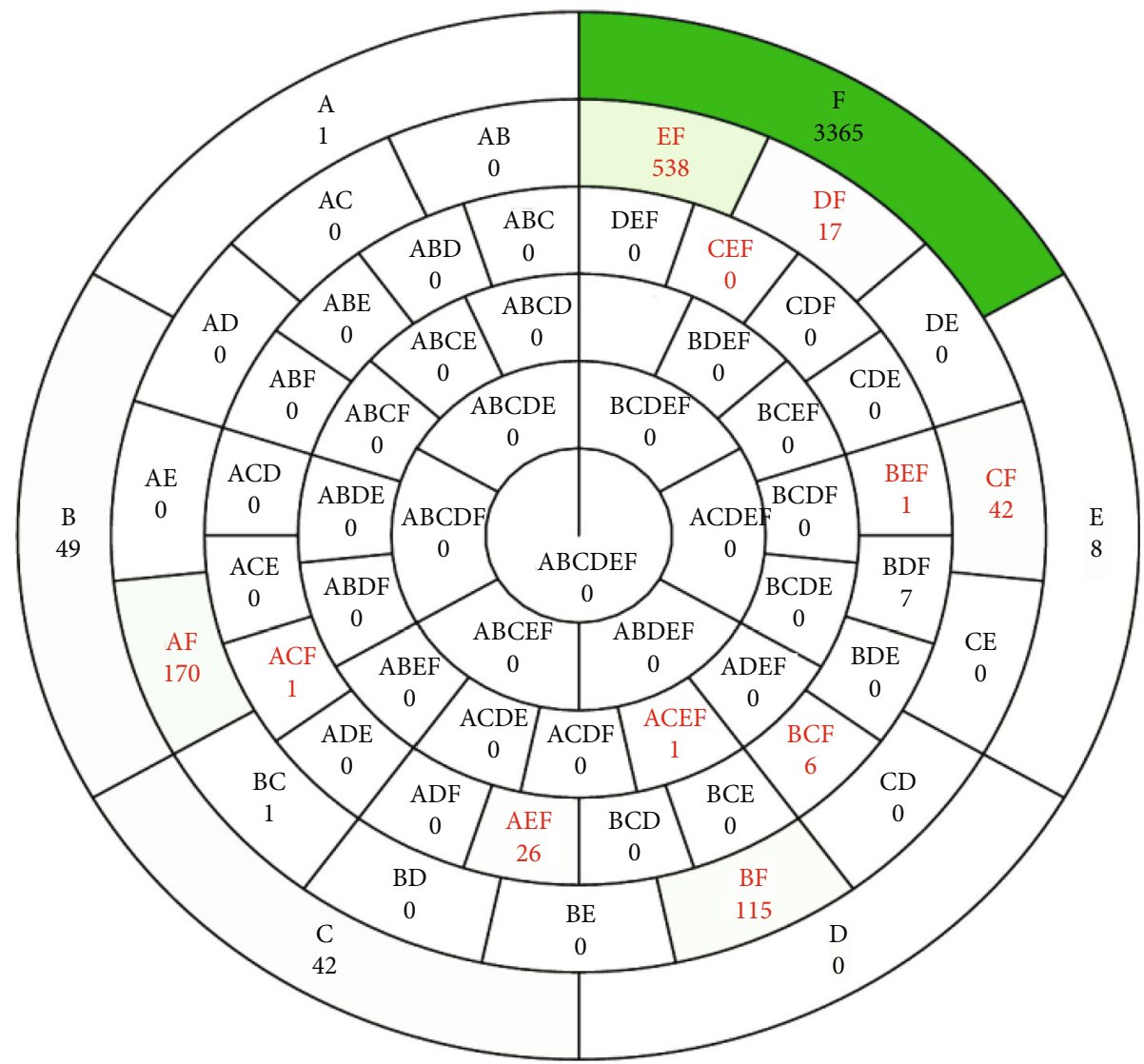

(a)

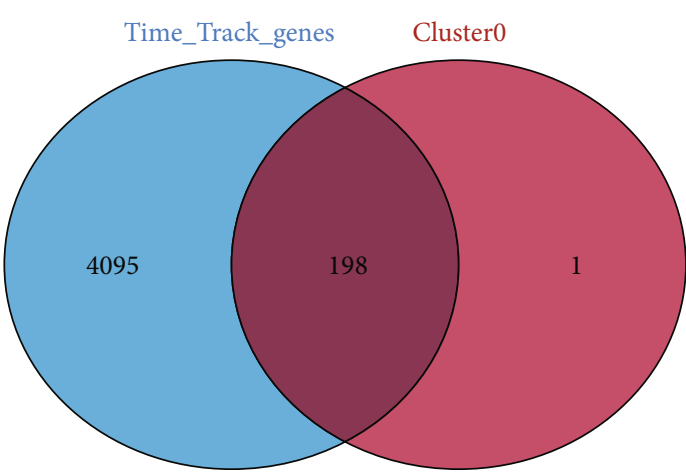

(b)

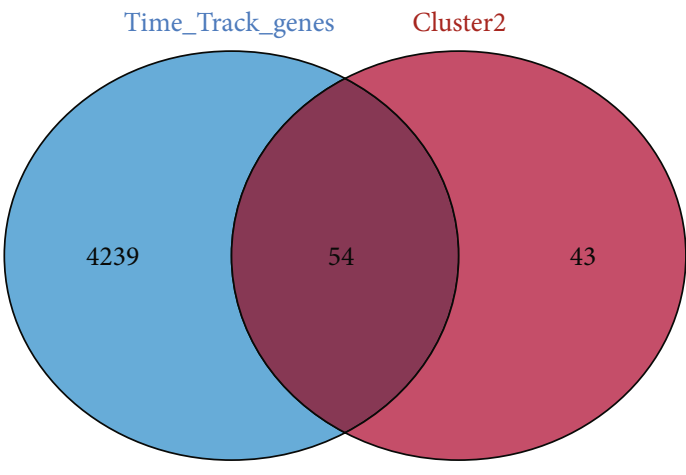

(d)

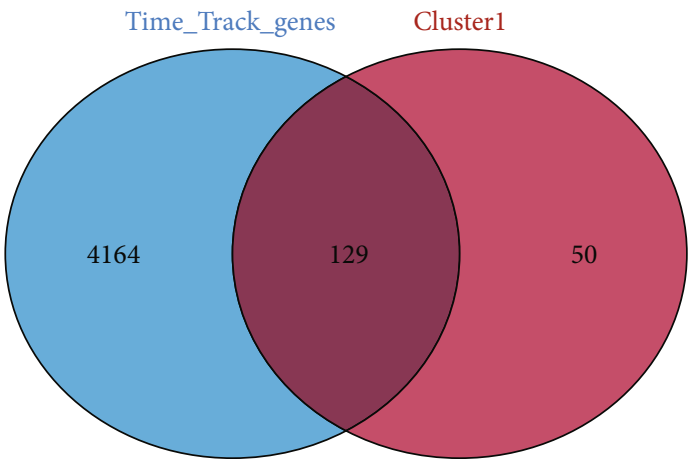

(c)

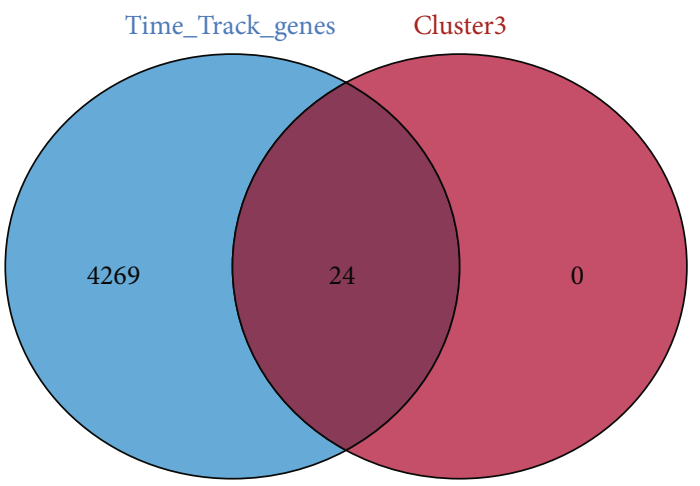

(e)

Figure 5: Continued. 


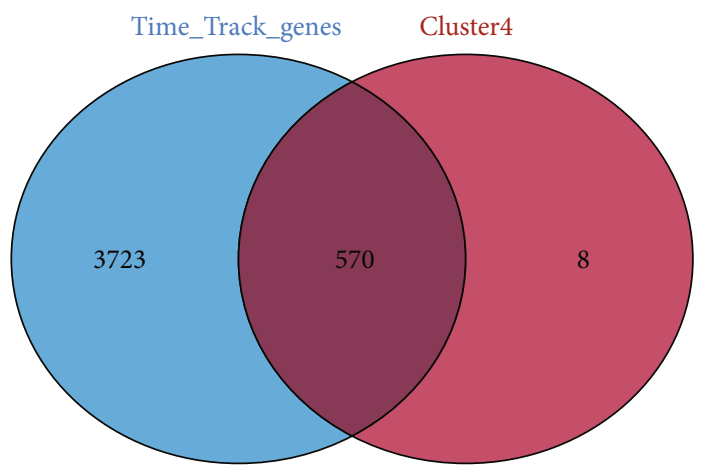

(f)

FIgURE 5: Intersection analysis of marker mRNAs and time track mRNAs for different clusters. (a) Total plot showing the intersection of different clustered marker mRNAs and time track mRNAs. Each compartment shows the cluster and the number of mRNAs variably contained within it (A: cluster 1; B: cluster 2; C: cluster 3; D: cluster 4; E: pseudotime track-related gene cluster). (b-f) Venn diagram of marker mRNAs and time track mRNAs for the different clusters.

clusters with $p$ values less than 0.001 (Figures $1(\mathrm{c})$ and $1(\mathrm{~d})$ ). UMAP analysis was then conducted, where five clusters were identified for further screening (Figure 1(e)). Ultimately, the singleR package was used to annotate these five clusters, confirming that all these cells were neural cells (Figure 1(f)).

\subsection{Single-Cell Analysis Reveals the Heterogeneity of Neural} Cells in the Neonatal Mouse Spinal Cord. The data from the scRNA-seq dataset was collected from neonatal mouse cervical and lumbar spine interneurons, which were sorted via flow cytometry. Based on data from previous studies, a UMAP plot was created to show the expression of the following markers: NFIB, ZFHX3, SHOX2, NEUROD2, SP8, and LHX3 (Figure 2(a)) [8]. In this study, 949 cells were screened by Seurat package for R software and were grouped into 5 clusters (Figure 2(b)). Among these cells, ZFHX3 and SHOX2 were jointly enriched in clusters 0,2 , and 4 , while NFIB, NEUROD2, and SP8 were jointly enriched in clusters 1 and 3 . As shown in the figure, the top 10 marker mRNAs for each cell cluster are shown in the heat map. Nefl, Zfhx3, Zfhx4, Vamp1, Nrn1, Vstm2a, Slc8a1, Thy1, Nxph4, and Slc12a5 are the marker mRNAs for cluster 0; Tcf4, Nfib, Neurod2, Ebf1, Satb1, Nfix, Zeb2, Hoxb8, Ass1, and Sp8 are the marker mRNAs for cluster 1; Scg2, Glt8d1, Calb2, Ecel1, Mpped2, Fos, Dlk1, Junb, Dlk1, Junb, Pcdh17, and Shox2 are the marker mRNAs for cluster 2; Ceacam8, Ceacam7, Psg7, Ceacam6, Psg5, Psg1, Ceacam1, Ceacam5, Psg11, Psg2, Psg8, Psg3, Psg9, Ceacam3, Psg6, and Psg3 are the marker mRNAs for cluster 3; Ppia, Cox5a, Calm1, Atp5mc3, Rabac1, Mgst3, Ubb, Myl12b, Ndufb6, and Atp6voc are the marker mRNAs for cluster 4 (Figure 2(c)). We identified the Psgs and the Ceacams as important marker mRNAs for cluster 3 . In the present study, spinal cord neuronal cells from newborn mice were subjected to mimetic chronological analysis on the UMAP map (Figure 2(d)). Cluster 3 was found to be at the final stage of the proposed chronological differentiation.

3.3. Psgs and Ceacams Are Specifically Expressed in Cluster 3. The UMAP plot shows the expression of all marker mRNAs in neonatal mouse spinal cord neuronal cells (Figure 3). Psgs and Ceacams were found to be specifically expressed in cluster 3. Marker mRNAs with pseudotiming analysis was shown in Figure 4. Marker mRNAs in cluster 3 (Ceacam8, Ceacam7, Psg7, Ceacam6, Psg5, Psg1, Ceacam1, Ceacam5, Psg11, Psg2, Psg8, Psg3, Psg9, Ceacam3, Psg6, and Psg3) began only at the tail end of the pseudotemporal differentiation. This observation suggests that Psgs and Ceacams may be markers of neural maturation. In addition, we also found that the expression of the genes Atp5mc3, Atp6v0c, Calm1, Cox5a, Mgst3, Myl12b, Ndufb6, and Ppia increased with increasing chronology during the process of neuronal differentiation in mice. These genes may be potential biomarkers for the degree of differentiation of mouse spinal cord neuronal cells.

3.4. Acquisition of Markers Related to Pseudotiming Traces. To further elucidate the cell differentiation trajectories of various biomarkers, we conducted an intersection analysis. The general plot of the intersection analysis of marker mRNAs and time track mRNAs for the different clusters is shown in Figure 5(a). There are 198, 129, 54, 24, and 570 marker mRNAs associated with pseudotemporal trajectories in clusters $0,1,2,3$, and 4, respectively (Figures 5(b)-5(f)).

3.5. Functional Analysis of Pseudotemporal TrajectoryRelated Marker mRNAs. Enrichment analysis of the 198 marker genes in cluster 0 showed that the functions of these genes were significantly enriched in the modulation of the chemical synaptic transmission, regulation of transsynaptic signaling, neurotransmitter secretion, axon part, presynapse, postsynaptic specialization, structural constituent of cytoskeleton, calmodulin binding, metal ion transmembrane transporter activity, endocrine and other factor-regulated calcium, reabsorption axon guidance, and prion disease (Figure 6(a)). The PPI network for these genes is shown in Figure 6(b). The enrichment analysis of the 129 marker genes in cluster 1 showed that the functions of these genes were enriched in response to the presence of ammonium ion, embryonic organ morphogenesis, anterior/posterior pattern specification, regionalization, nuclear transcription 

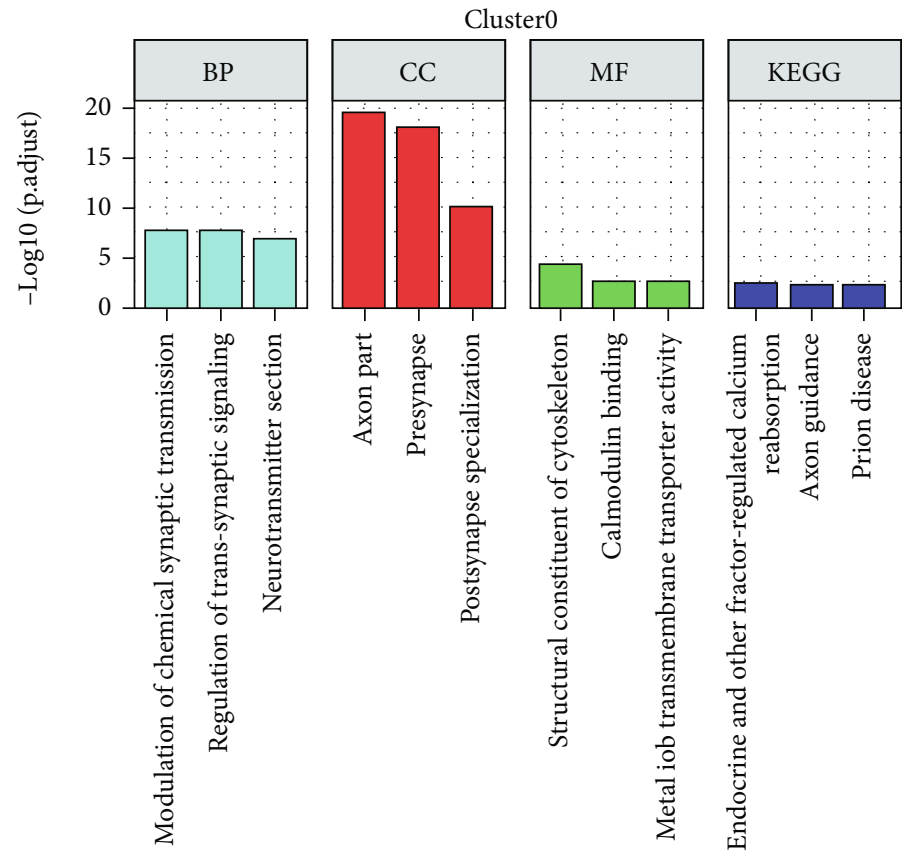
$\square$ BP
$\square \mathrm{MF}$
$\square$ CC
$\square$ KEGG

(a)

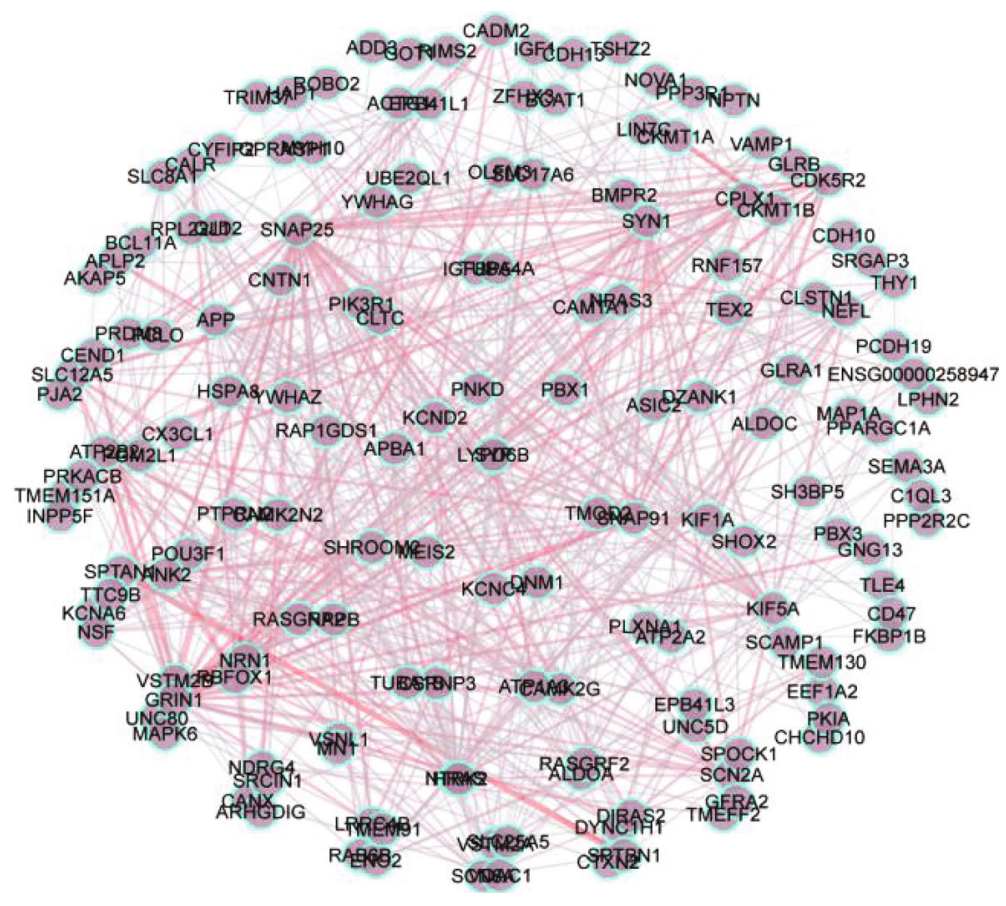

(b)

FIgure 6: Continued. 

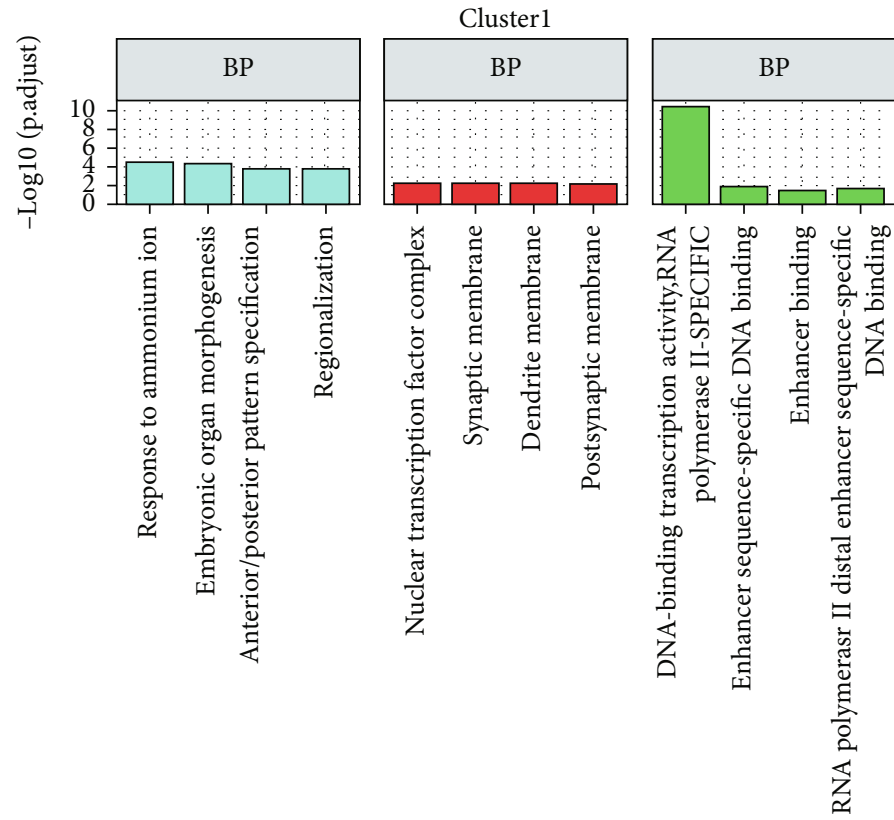

$$
\begin{aligned}
& \square \text { BР } \\
& \square \text { CC } \\
& \square \text { MF }
\end{aligned}
$$

(c)

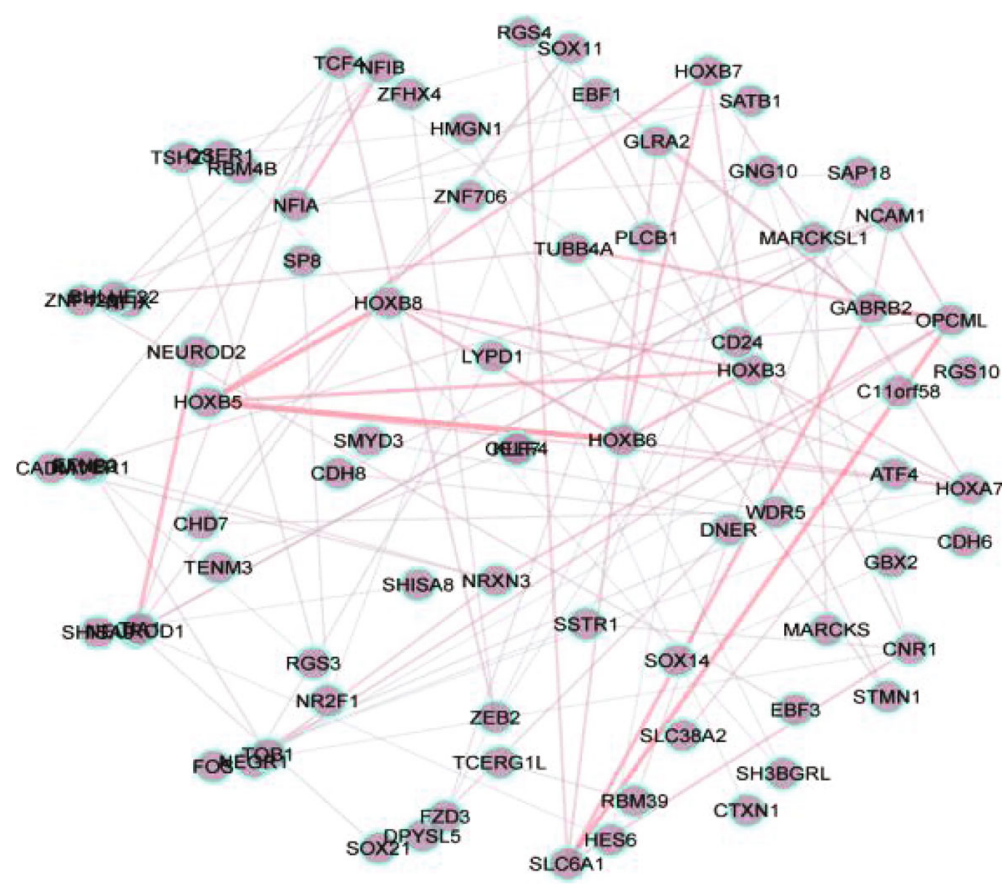

(d)

Figure 6: Continued. 

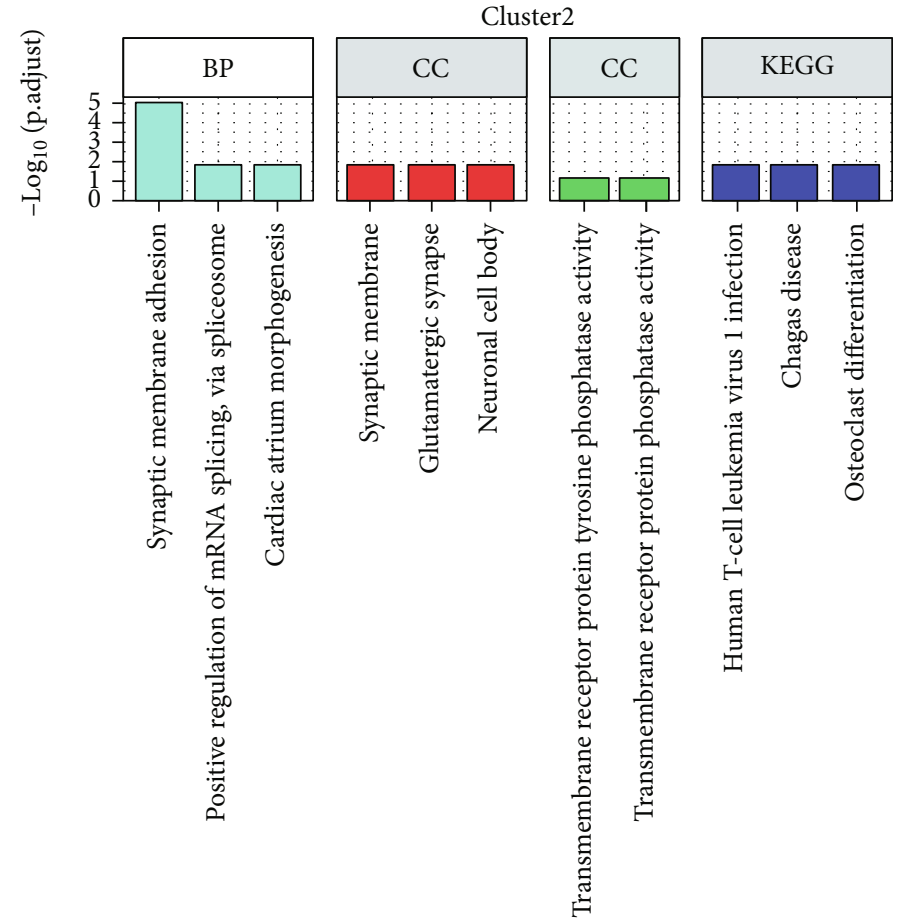
$\square$ BP
$\mathrm{MF}$
$\square$ CC
KEGG

(e)

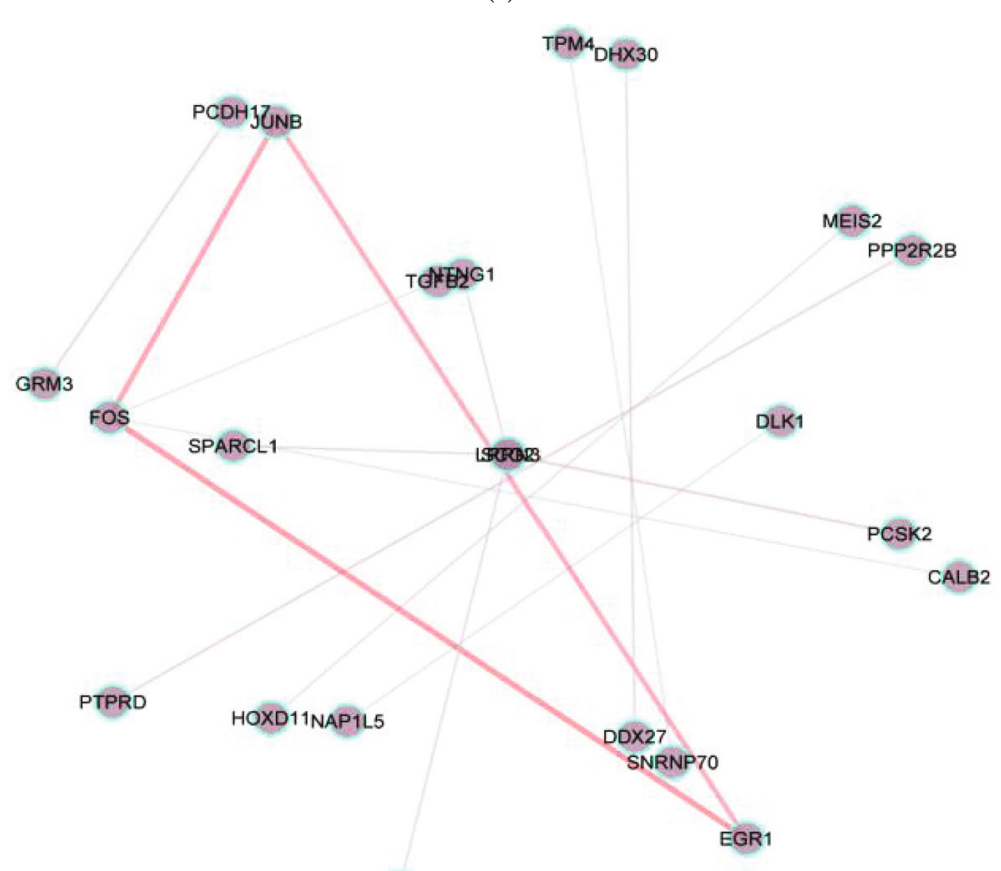

ADAM22

(f)

FIGURE 6: Enrichment analysis of clusters 0-2 and the corresponding PPI network analysis. (a, b) Enrichment analysis and PPI network of the 198 intersecting mRNAs in cluster 0. (c, d) Enrichment analysis of the 129 intersecting mRNAs and PPI network of cluster 1. (e, f) Enrichment analysis of the 54 intersecting mRNAs and PPI network of cluster 2. 


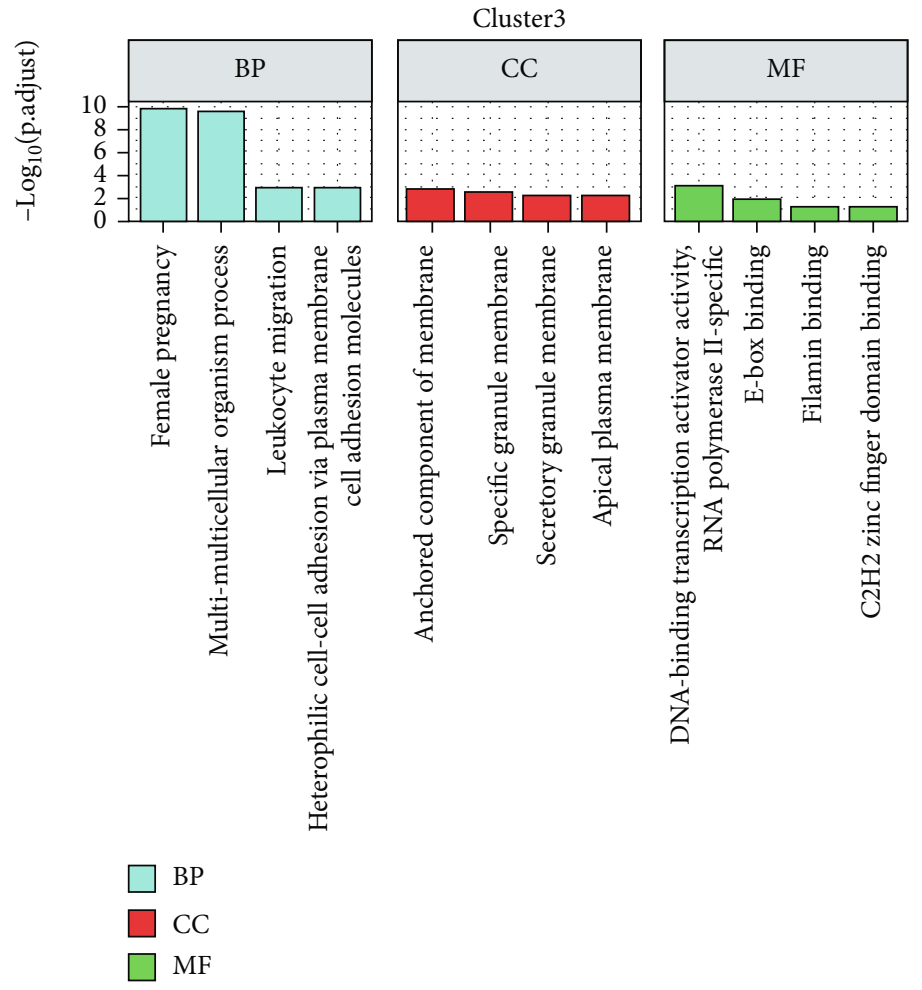

(a)

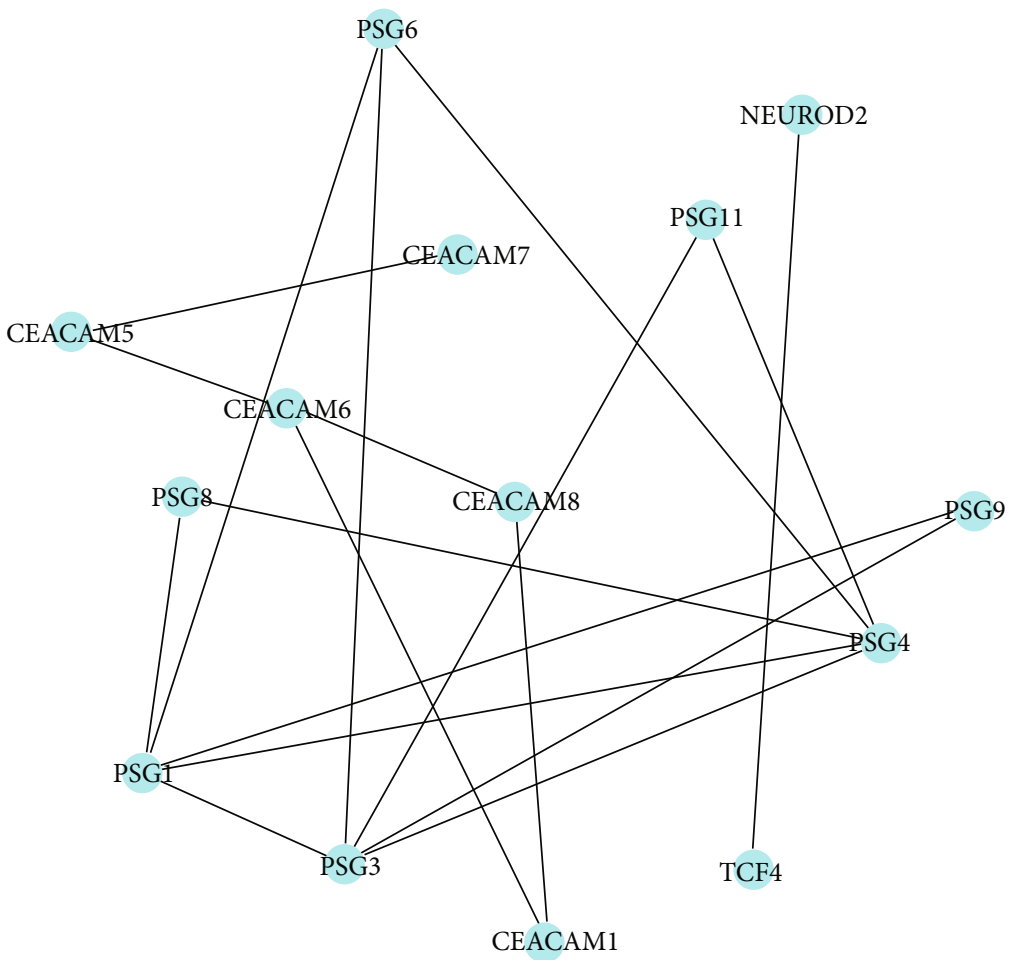

(b)

FIgUre 7: Continued. 


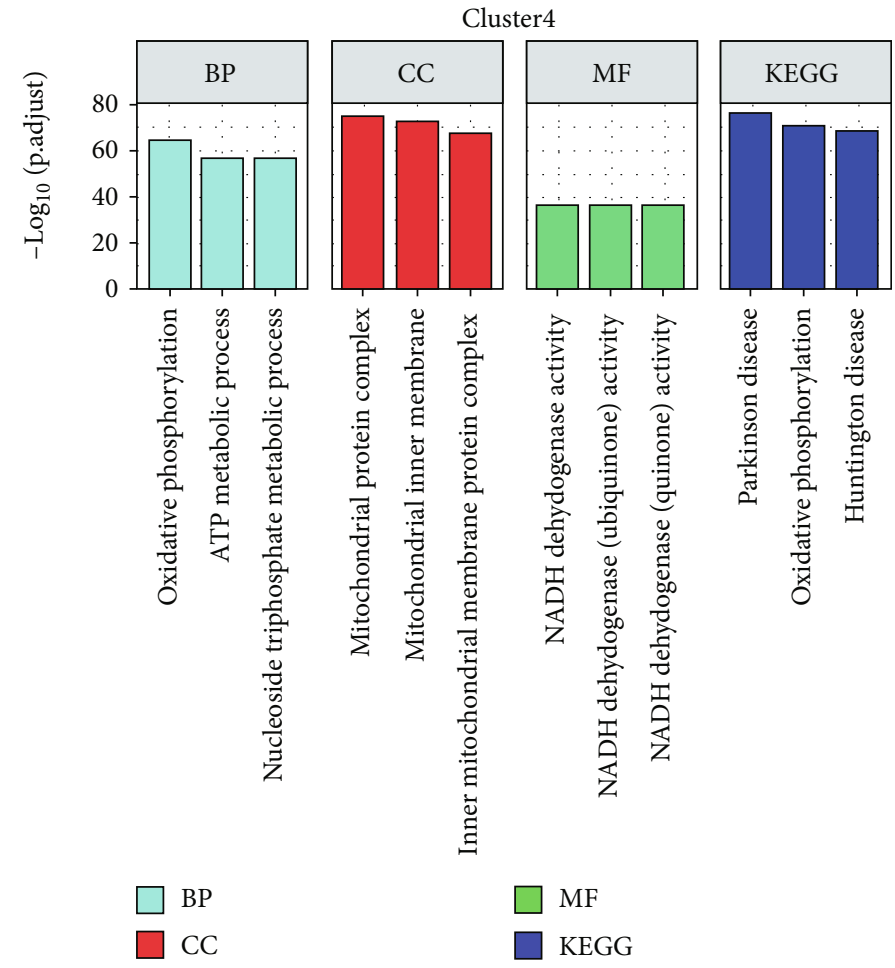

(c)

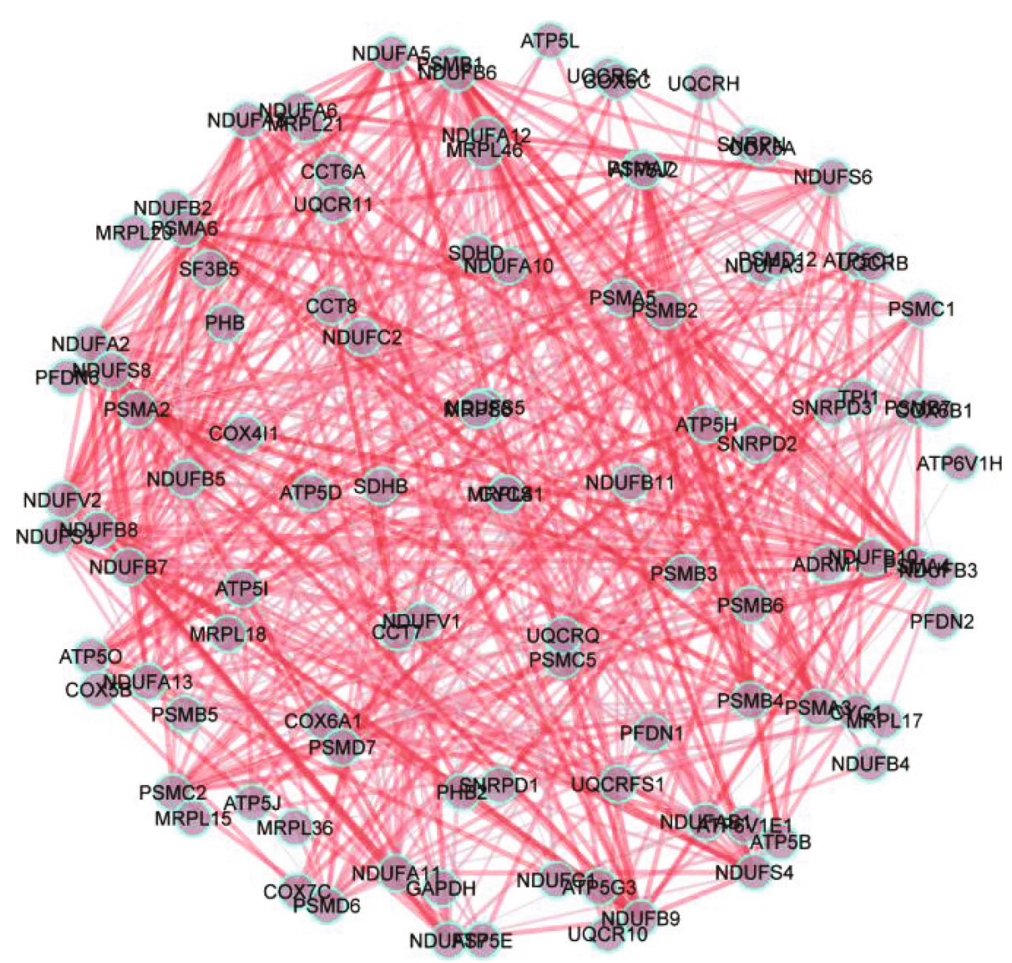

(d)

FIGURE 7: Enrichment analysis of clusters 3 and 4 and their corresponding PPI network analysis. (a, b) Enrichment analysis and PPI network of the 24 intersecting genes in cluster 3. (c, d) Enrichment analysis and PPI network of the 570 intersecting genes in cluster 4.

factor complex, synaptic membrane, dendrite membrane, postsynaptic membrane, DNA-binding transcription activator activity, enhancer sequence-specific DNA binding, enhancer binding, and RNA polymerase II distal enhancer sequence-specific DNA binding (Figure 6(c)). The PPI network for these genes is presented in Figure 6(d).

Enrichment analysis of the 54 marker genes in cluster 2 showed that the functions of these genes were significantly 


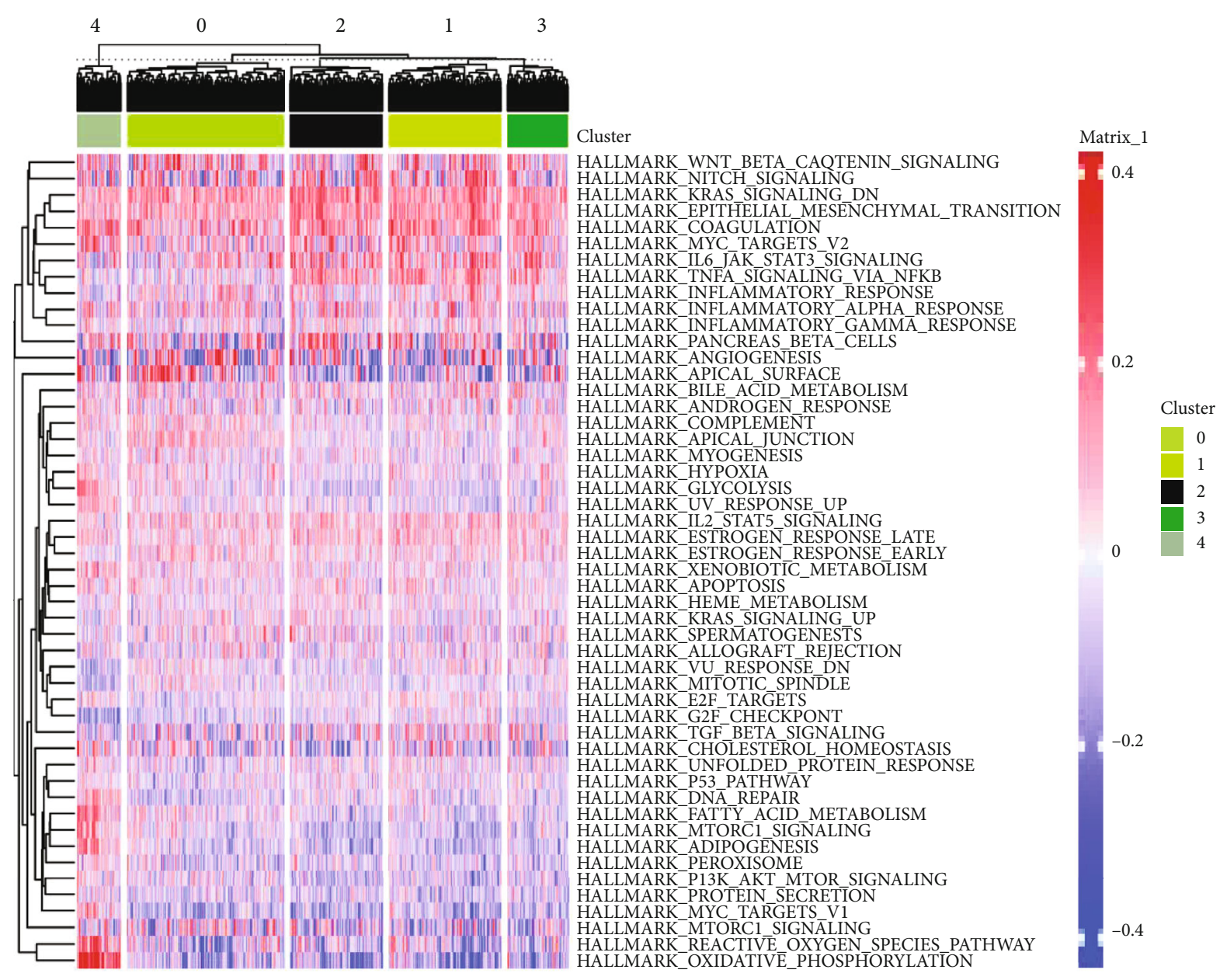

FIGURE 8: The pathways associated with each cluster as obtained via pathway enrichment analysis.

enriched during synaptic membrane adhesion, positive regulation of mRNA splicing-via the spliceosome, cardiac atrium morphogenesis, synaptic membrane, glutamatergic synapse, neuronal cell body, transmembrane receptor protein tyrosine phosphatase activity, human T-cell leukemia virus 1 infection, Chagas disease, and osteoclast differentiation (Figure 6(e)). The PPI network for these genes is presented in Figure 6(f).

Enrichment analysis of the 24 marker genes in cluster 3 showed that these genes were significantly enriched during female pregnancy, multicellular organism process, leukocyte migration, and heterophilic cell-cell adhesion via plasma membrane cell adhesion molecules (Figure 7(a)). The PPI network for these genes is presented in Figure 7(b). Psgs and Ceacams were found to be the core genes of this PPI network.

Enrichment analysis of the 270 marker mRNAs in cluster 4 showed that their functions were significantly enriched in oxidative phosphorylation, ATP metabolic process, nucleoside triphosphate metabolic process, mitochondrial protein complex, mitochondrial inner membrane, inner mitochondrial membrane protein complex, NADH dehydrogenase activity, Parkinson disease, oxidative phosphorylation, and Huntington disease (Figure $7(\mathrm{c})$ ). The PPI network for these genes is shown in Figure $7(\mathrm{~d})$. Finally, GSVA enrichment analysis demonstrated the differential expression of the different pathways in the Reactome database across the different clusters (Figure 8).

3.6. Identification of Transcriptional Regulatory Networks Associated with USF2/CEACAM1\&5 and KLF6/PSG3\&5. Psgs and Ceacames were found to be the core genes for cluster 3 in this study. The TRRUST database was then used to find upstream transcriptional regulatory targets for Psgs and Ceacams. The transcriptional network of PSGs and CEACAMs were shown in Figure 9(a), wherein the upstream transcriptional regulatory targets of CEACAM1, CEACAM5, PSG1, PSG3, PSG5, and PSG7 were predicted. HNF4A, IRF1, NFKB1, RELA, SOX9, SP2, USF1, and USF2 were predicted to be transcriptional regulators of $C E A C A M 1$ and CEACAM5. FOXF2, KLF6, and MYBL2 were predicted to be transcriptional regulators of PSGs such as PSG1, PSG3, PSG5, and PSG7. Correlation analysis suggested a significant correlation between PSGs and CEACAMs in the GSE108788 dataset (Figure 9(a)). It was also observed that CEACAM1 and CEACAM5 were significantly positively correlated with the expression of USF2 (Figure 9(b)). Similarly, PSG3 and PSG5 were significantly positively correlated with 


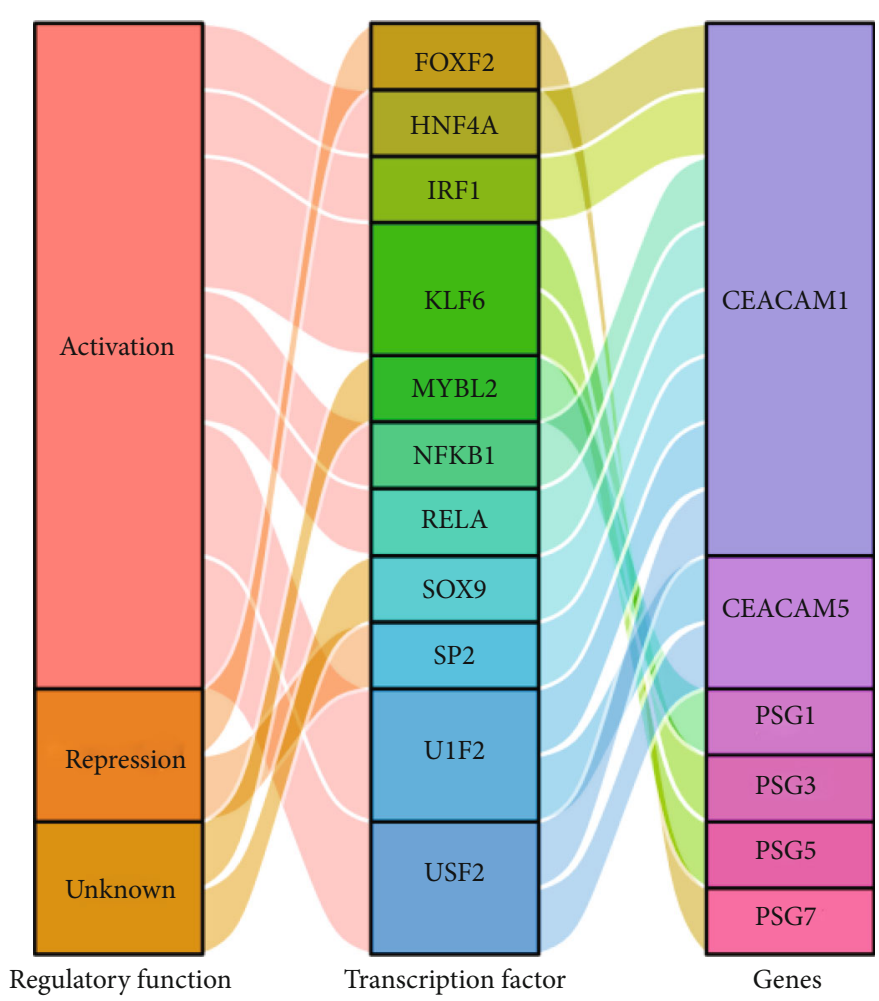

(a)

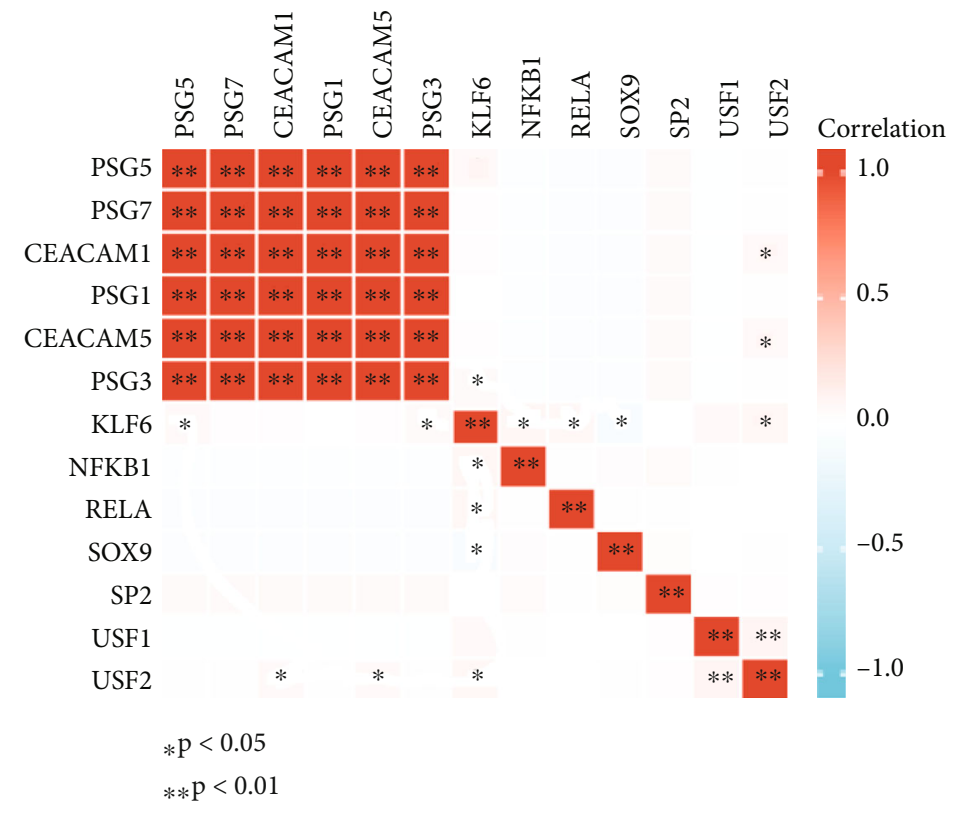

(b)

Figure 9: Continued. 

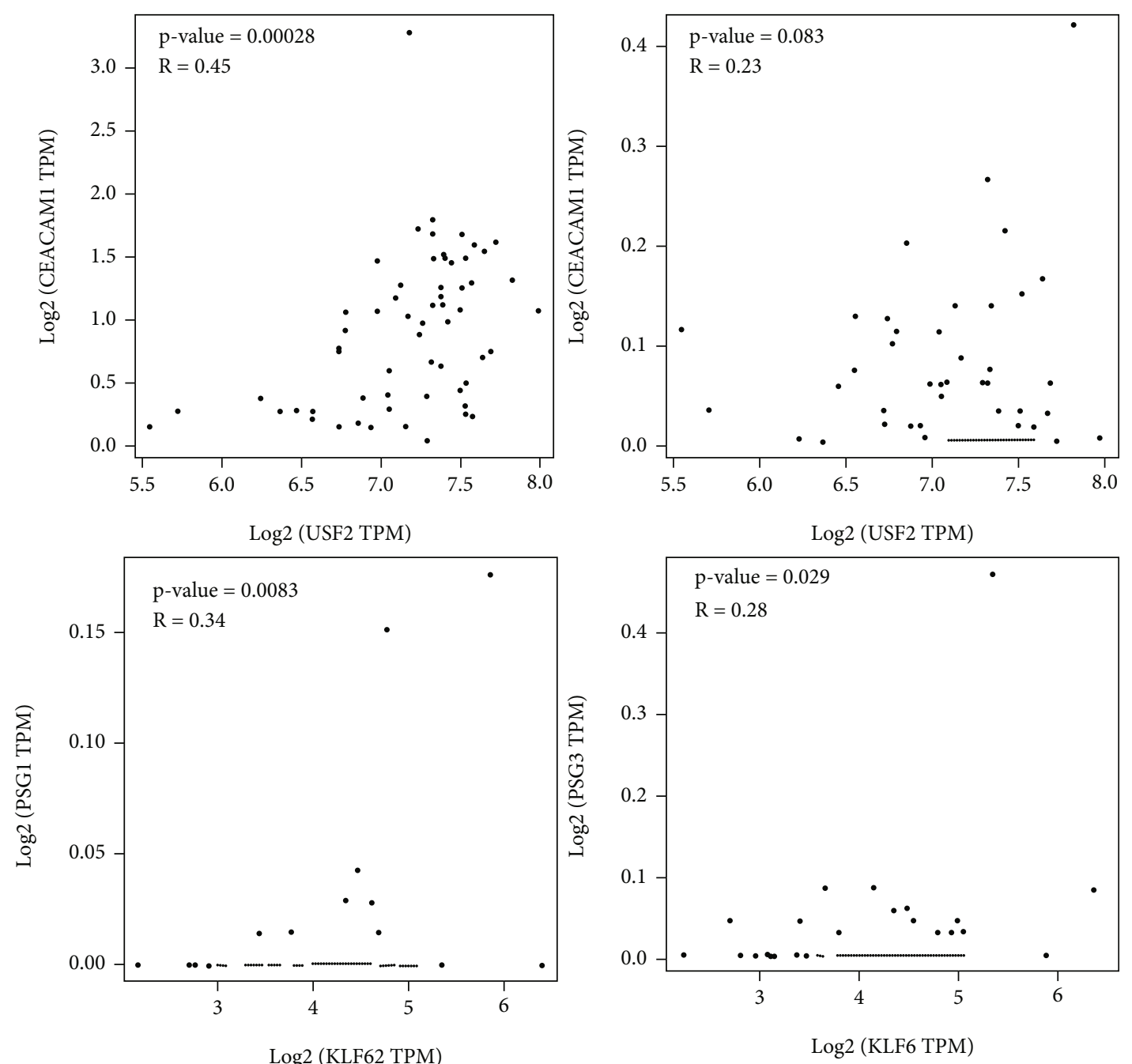

$\log 2($ KLF62 TPM)

(c)

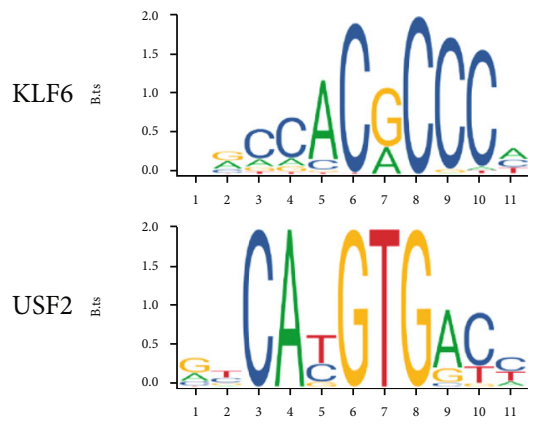

(d)

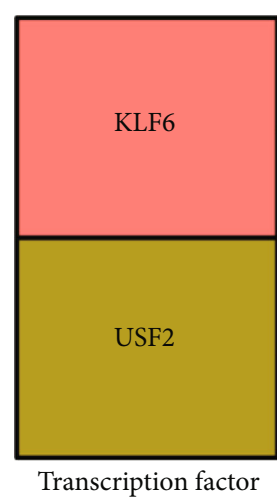

Transcription factor

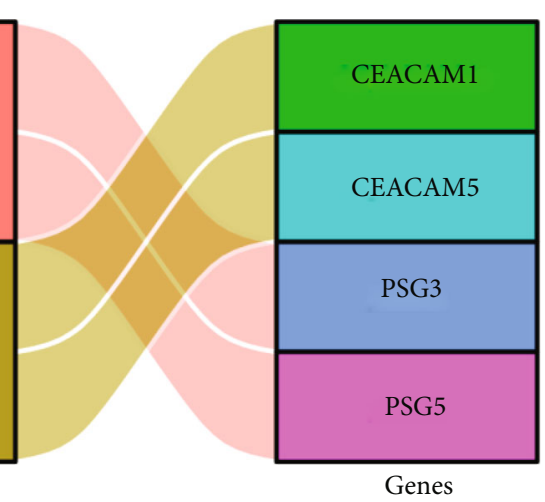

(e)

FIGURE 9: Identification of USF2/CEACAM1\&5 and KLF6/PSG3\&5 transcriptional regulatory networks. (a) Transcriptional networks of Psgs and Ceacams. (b) Correlation analysis of Psgs, Ceacams, and their potential transcriptional regulators. (c) Correlation analysis based on the GTEX dataset. (d) Motif loci for KLF6 and USF2. (e) Identification of USF2/CEACAM1\&5 and KLF6/PSG3\&5 transcriptional regulatory networks.

the expression of KLF6 (Figure 9(b)). However, GSE108788based correlation analysis of scRNA-seq dataset found that the results for FOXF2, HNF4A, IRF1, and MYBL2 did not match the results expected in Figure 9(a). The Genotype-
Tissue Expression (GTEx) project is a dataset that reflects the relationship between genetic variants and gene expression in human tissues. In spinal cord samples from the GTEx dataset, KLF6 expression was found to have a positive 


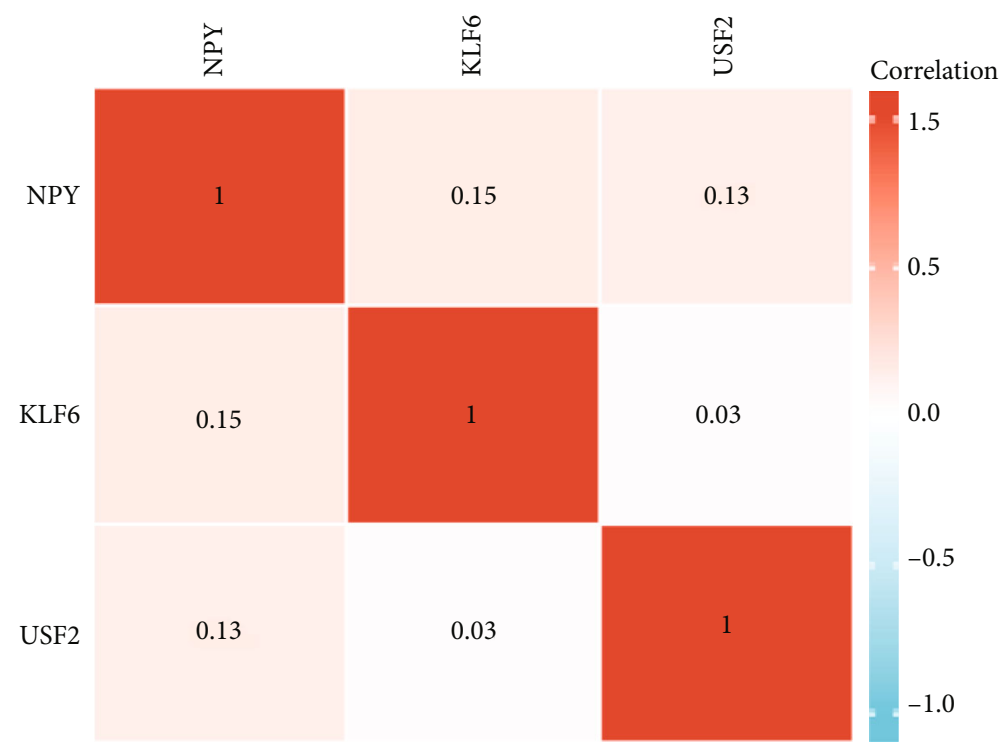

(a)

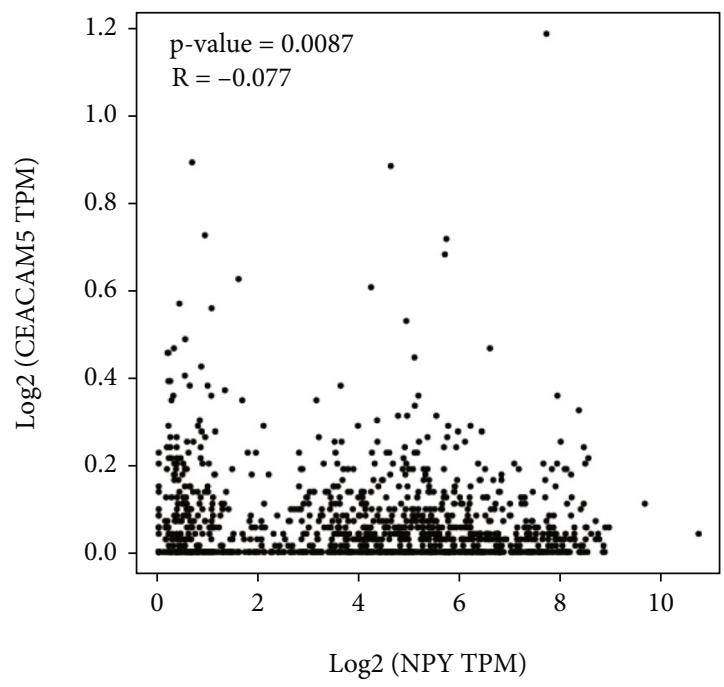

(c)

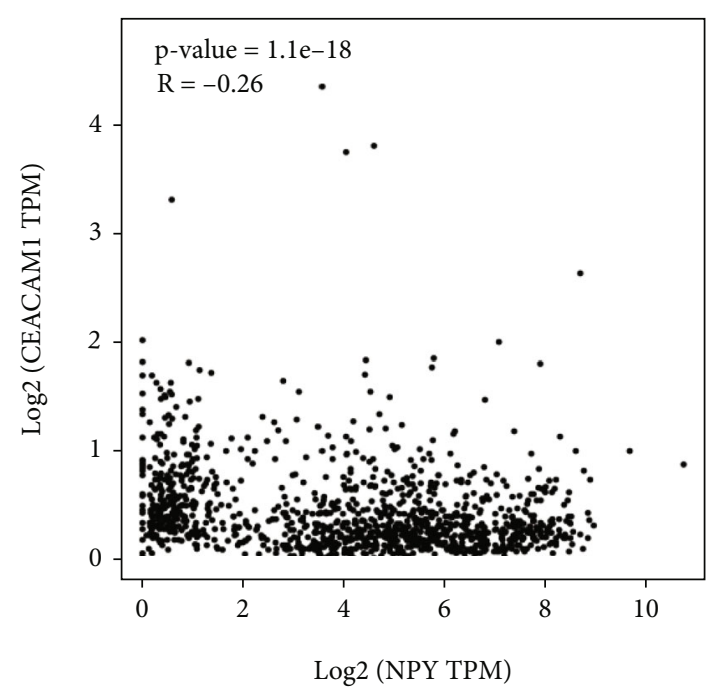

(b)

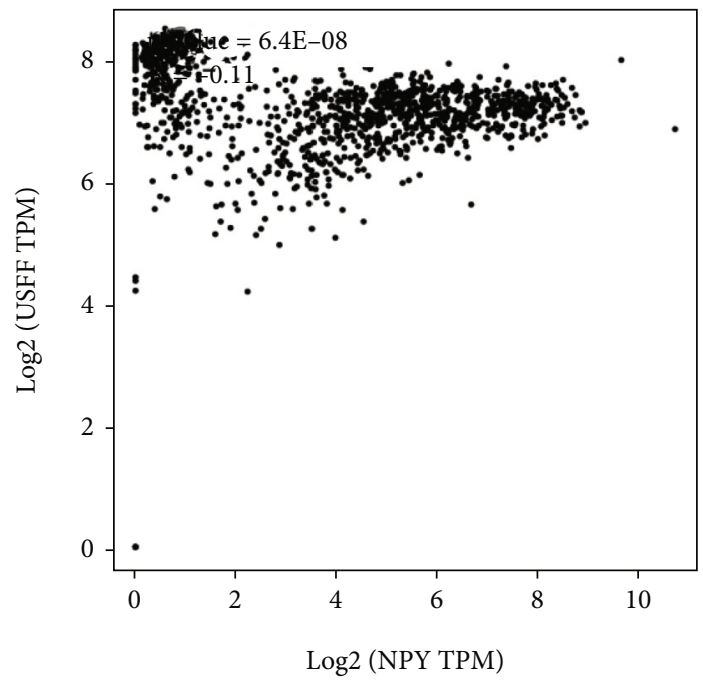

(d)

FIGURE 10: The spinal cord developmental marker NPY and its association with CEACAM1, CEACAM5, and USF2. (a) Correlation analysis of NPY, KLF6, and USF2. (b) Correlation analysis of NPY and CEACAM1. (c) Correlation analysis of NPY and CEACAM5. (d) Correlation analysis of NPY and USF2.

correlation with the expression of PSG1 and PSG3, while USF2 expression was found to have a significant positive correlation with the expression of CEACAM5. The binding sites of the motifs of KLF6 and USF2 are shown in Figure 9(d) based on the TRRUST database. Based on these results, USF2 was presumed to be a transcriptional regulator of CEACAM1 and CEACAM5 while KLF6 is presumed to be a transcriptional regulator of PSG3 and PSG5 (Figure 9(e)). The spinal cord developmental marker NPY was positively correlated with CEACAM1, CEACAM5, and USF2 (Figures 10(a)-10(d)).

\section{Discussion}

In this study, single-cell analysis was utilized to reveal the heterogeneity and pseudotemporal differentiation trajectory analysis of the neonatal mouse spinal cord neuronal cells. Psgs and Ceacams were found to be the core genes for cluster 3 , where the marker genes in these cell subpopulations characterize the transcriptional profile of mouse spinal cord neuronal cells.

Our results show that the molecular diversity of cell types plays an important role in the development of the mouse spinal cord, similar to previous studies that conducted large-scale molecular profiling [18-21]. The spinal cord nerve cells of mice were divided into five clusters. These results provide new insights into the temporal patterns of gene expression, thus establishing a new framework for the analysis of the mouse spinal cord.

This study provides a clustering map for the clustering of different cells correlated to their function. The cluster 0 
marker genes were functionally enriched mainly in synapses with signaling; the marker genes in cluster 1 are mainly enriched in gene transcription; the marker genes in cluster 2 are mainly enriched in transmembrane transport and cellular communication in neuronal cells; the marker genes in cluster 3 are remarkably enriched in female pregnancy and multicellular organism processes; and the marker genes in cluster 4 are mainly related to energy metabolism and some disease pathways including Parkinson's disease, oxidative phosphorylation, and Huntington's disease. These results suggest that the differential enrichment of genes in different cells implies the functional differences in the corresponding regional tissues [22].

The Psg family genes and the Ceacam family genes were found to be the core genes for cluster 3 in this study. Cluster 3 were found to be remarkably enriched in female pregnancy and multicellular organism processes. The pregnancyspecific glycoprotein (PSG) gene is a member of the carcinoembryonic antigen cell adhesion molecule (CEACAM) family genes [23]. Both Psg and Ceacam family genes play a key role in tumor progression [24]. In mammals, CEACAMs and PSGs are involved in feto-maternal interactions. However, previous studies have not systematically investigated the developmental trajectories of these two groups of genes in humans [25]. In addition, CEACAMs and PSGs have been found play a prominent role in the study of species evolution [25, 26], as the expression of PSG may imply the adaptive evolution of species [27]. Our study revealed that PSGs and CEACAMs are potentially involved in neural development.

KLF6, a zinc-finger transcription factor of the KLF family, is involved in several processes including cell development, differentiation, and regulation [28]. KLF6 was found to be a key transcription factor involved in the central neuro-mediated apoptosis with the function of reducing neurological damage after cerebral hemorrhage [29]. Based on bioinformatic analysis, KLF6 was found to be a potential transcriptional regulator for PSG3 and PSG5 in this study. The upstream stimulatory factors (USFs) USF1 and USF2 all belong to the helix-loop-helix leucine zipper transcription factor family and function as homodimers or heterodimers by binding to the e-box of the target DNA core sequence $\left(5^{\prime}\right.$-CANNTG- $\left.3^{\prime}\right)[30,31]$. USFs play an important role in stress, the immune response, energy metabolism, and cell development [32-34]. The role of USF2 seems to be more critical than that of USF1 $[35,36]$. In this study, USF2 was hypothesized to be a transcriptional regulator of CEACAM1 and CEACAM5.

Neuropeptide Y (NPY) was discovered by Tatemoto et al. in 1982 and was found to be localized in the nervous system; its functional role has been intensively investigated [37]. Previous studies suggested that NPY may function as a neurotransmitter, has a neuromodulatory function, or has neuroendocrine function [36, 38-40]. Although the morphology and distribution of NPY in the adult spinal cord have been reported, the development of NPY during the formation of the human fetal spinal cord and its patterns have not yet been reported in the literature [36, 38, 39]. In this study, the spinal cord developmental marker NPY was found to be positively correlated with CEACAM1, CEACAM5, and USF2.

Advances in single-cell transcriptomics have allowed us to gain groundbreaking insights into the heterogeneous patterns of gene expression over time in the development of spinal nerves. The heterogeneity of neonatal mouse spinal nerve cells was revealed in this study through single-cell analysis. The spinal cord nerve cells of mice were divided into five clusters, giving an initial indication of the complexity of the differential expression of the genes of these cells over time. These results provide new insights into the temporal patterns of gene expression, thus establishing a new framework for the analysis of the mouse spinal cord. Psgs and Ceacams were also defined for the first time in this study as marker mRNAs clustered within the same cell. The upstream transcriptional regulators of the Psg and Ceacam genes were also identified. In future studies, immunohistochemical experiments, as well as in vivo and in vitro studies will still be needed to validate our results.

\section{Conclusions}

In summary, we analyzed recently published single-cell datasets and defined five subgroups of marker mRNAs that can be used for future single-cell transcriptome analyses. This study also revealed that PSGs and CEACAMs are potentially involved in neural development. Identifying these transcriptional regulatory networks will allow us to investigate further the developmental trajectory of mouse neurons and the cell biology of diseases.

\section{Abbreviations}

ANOVA: Analysis of variance

CEACAM: Carcinoembryonic antigen-related cell adhesion molecule

CNS: Central nervous system

GEO: Gene Expression Omnibus

GO: $\quad$ Gene Oncology

GTEX: The Genotype-Tissue Expression

GSVA: Gene set variation analysis

KEGG: $\quad$ Kyoto Encyclopedia of Genes and Genomes

PPI: $\quad$ Protein-protein interaction network

PSG: $\quad$ Pregnancy-specific glycoproteins.

\section{Data Availability}

All original data for this study were obtained from the GSE131882 dataset in the GEO database and the Genotype-Tissue Expression (GTEx) project.

\section{Conflicts of Interest}

The authors declare that they have no conflicts of interest.

\section{Authors' Contributions}

$\mathrm{XL}, \mathrm{WL}$, and WG came up with and designed the experiments. XL, WL, and WG conducted the experiments although all authors analyzed the data. In addition, XL, 
$\mathrm{WL}$, and WG contributed the reagents/materials/analysis tools. XL, WL, and WG wrote the manuscript. All authors contributed to the article and approved the submitted version. Xinbing Liu and Wei Gao contributed equally to this work.

\section{Acknowledgments}

We thank all the anonymous reviewers for their pertinent suggestions for this study. The study received funding support from the Changxing People's Hospital.

\section{References}

[1] A. Sagner and J. Briscoe, "Establishing neuronal diversity in the spinal cord: a time and a place," Development, vol. 146, no. 22, article dev182154, 2019.

[2] F. Homayouni, A. Abbasi, A. Vakili-Zarch, M. Mansouri, M. Babaei-Zarch, and M. Hossein, "Direct remyelinating effect of bone marrow mesenchymal stem cells in the rat model of spinal cord injury," Life Research, vol. 4, no. 3, p. 1, 2021.

[3] C. D. Stern, "Initial patterning of the central nervous system: how many organizers?," Nature Reviews Neuroscience, vol. 2, no. 2, pp. 92-98, 2001.

[4] C. D. Stern, "Neural induction: 10 years on since the 'default model'," Current Opinion in Cell Biology, vol. 18, no. 6, pp. 692-697, 2006.

[5] X. Kang, Y. Chen, B. Yi et al., "An integrative microenvironment approach for laryngeal carcinoma: the role of immune/ methylation/autophagy signatures on disease clinical prognosis and single-cell genotypes," Journal of Cancer, vol. 12, no. 14, pp. 4148-4171, 2021.

[6] J. Wu, J. Qin, L. Li et al., "Roles of the immune/methylation/ autophagy landscape on single-cell genotypes and stroke risk in breast cancer microenvironment," Oxidative Medicine and Cellular Longevity, vol. 2021, 32 pages, 2021.

[7] Z. Zeng, N. Miao, and T. Sun, "Revealing cellular and molecular complexity of the central nervous system using single cell sequencing," Stem Cell Research \& Therapy, vol. 9, no. 1, p. 234, 2018.

[8] M. Hayashi, C. A. Hinckley, S. P. Driscoll et al., "Graded arrays of spinal and supraspinal V2a interneuron subtypes underlie forelimb and hindlimb motor control," Neuron, vol. 97, no. 4, pp. 869-884.e5, 2018.

[9] W.-W. Lin, L.-T. Xu, Y.-S. Chen, K. Go, C. Sun, and Y.-J. Zhu, "Single-cell transcriptomics-based study of transcriptional regulatory features in the mouse brain vasculature," BioMed Research International, vol. 2021, 15 pages, 2021.

[10] T. Stuart, A. Butler, P. Hoffman et al., "Comprehensive integration of single-cell data," Cell, vol. 177, no. 7, pp. 18881902.e21, 2019.

[11] D. Aran, A. P. Looney, L. Liu et al., "Reference-based analysis of lung single-cell sequencing reveals a transitional profibrotic macrophage," Nature Immunology, vol. 20, no. 2, pp. 163-172, 2019.

[12] D. Szklarczyk, A. L. Gable, D. Lyon et al., "STRING v11: protein-protein association networks with increased coverage, supporting functional discovery in genome-wide experimental datasets," Nucleic Acids Research, vol. 47, no. D1, pp. D607D613, 2019.

[13] Y. Chen, X. Kang, Z. Zhou et al., "MiR-1908/EXO1 and MiR203a/FOS, regulated by scd1, are associated with fracture risk and bone health in postmenopausal diabetic women," Aging, vol. 12, no. 10, pp. 9549-9584, 2020.

[14] G. Yu, L.-G. Wang, Y. Han, and Q.-Y. He, "clusterProfiler: an $\mathrm{R}$ package for comparing biological themes among gene clusters," Integrative Biology, vol. 16, no. 5, pp. 284-287, 2012.

[15] A. Fabregat, S. Jupe, L. Matthews et al., "The reactome pathway knowledgebase," Nucleic Acids Research, vol. 46, no. D1, pp. D649-D655, 2018.

[16] A. S. Kalluri, S. K. Vellarikkal, E. R. Edelman et al., "Single-cell analysis of the normal mouse aorta reveals functionally distinct endothelial cell populations," Circulation, vol. 140, no. 2, pp. 147-163, 2019.

[17] H. Han, J.-W. Cho, S. Lee et al., "TRRUST v2: an expanded reference database of human and mouse transcriptional regulatory interactions," Nucleic Acids Research, vol. 46, no. D1, pp. D380-D386, 2018.

[18] J. Delile, T. Rayon, M. Melchionda, A. Edwards, J. Briscoe, and A. Sagner, "Single cell transcriptomics reveals spatial and temporal dynamics of gene expression in the developing mouse spinal cord," Development, vol. 146, no. 12, article dev173807, 2019.

[19] X. Li, Y. Guo, S. Wei, J. Chen, and Y. Wu, "Research progress on the influence of opioids on fetal neurodevelopment during pregnancy," Life Research, vol. 3, no. 2, p. 68, 2020.

[20] A. A. Pollen, A. Bhaduri, M. G. Andrews et al., "Establishing cerebral organoids as models of human-specific brain evolution," Cell, vol. 176, no. 4, pp. 743-756.e17, 2019.

[21] A. Sathyamurthy, K. R. Johnson, K. J. E. Matson et al., "Massively parallel single nucleus transcriptional profiling defines spinal cord neurons and their activity during behavior," Cell Reports, vol. 22, no. 8, pp. 2216-2225, 2018.

[22] M. G. Sharoar, X. Hu, X.-M. Ma, X. Zhu, and R. Yan, "Sequential formation of different layers of dystrophic neurites in Alzheimer's brains," Molecular Psychiatry, vol. 24, no. 9, pp. 13691382, 2019.

[23] R. Kammerer and W. Zimmermann, "Coevolution of activating and inhibitory receptors within mammalian carcinoembryonic antigen families," BMC Biology, vol. 8, no. 1, p. 12, 2010.

[24] A. Houston, J. M. Williams, T. L. Rovis et al., "Pregnancy-specific glycoprotein expression in normal gastrointestinal tract and in tumors detected with novel monoclonal antibodies," mAbs, vol. 8, no. 3, pp. 491-500, 2016.

[25] C. L. Chang, J. Semyonov, P. J. Cheng et al., "Widespread divergence of the CEACAM/PSG genes in vertebrates and humans suggests sensitivity to selection," PLoS One, vol. 8, no. 4, article e61701, 2013.

[26] D. Aleksic, L. Blaschke, S. Mißbach et al., "Convergent evolution of pregnancy-specific glycoproteins in human and horse," Reproduction, vol. 152, no. 3, pp. 171-184, 2016.

[27] R. Kammerer, M. Mansfeld, J. Hänske et al., "Recent expansion and adaptive evolution of the carcinoembryonic antigen family in bats of the Yangochiroptera subgroup," BMC Genomics, vol. 18, no. 1, p. 717, 2017.

[28] S. Syafruddin, M. Mohtar, W. Wan Mohamad Nazarie, and T. Low, "Two sides of the same coin: the roles of KLF6 in physiology and pathophysiology," Biomolecules, vol. 10, no. 10, p. 1378, 2020.

[29] J. Sun, J. Cai, J. Chen et al., "Krüppel-like factor 6 silencing prevents oxidative stress and neurological dysfunction following 
intracerebral hemorrhage via sirtuin 5/Nrf2/HO-1 axis," Frontiers in Aging Neuroscience, vol. 13, article 646729, 2021.

[30] M. Sawadogo, "Interaction of a gene-specific transcription factor with the adenovirus major late promoter upstream of the TATA box region," Cell, vol. 43, no. 1, pp. 165-175, 1985.

[31] B. Viollet, A.-M. Lefrançois-Martinez, A. Henrion, A. Kahn, M. Raymondjean, and A. Martinez, "Immunochemical characterization and transacting properties of upstream stimulatory factor isoforms (*)," Journal of Biological Chemistry, vol. 271, no. 3, pp. 1405-1415, 1996.

[32] T. F. Chi, F. Khoder-Agha, D. Mennerich et al., "Loss of USF2 promotes proliferation, migration and mitophagy in a redoxdependent manner," Redox Biology, vol. 37, article 101750, 2020.

[33] S. Corre and M.-D. Galibert, "USF : un régulateur essentiel de la transcription," médecine/sciences, vol. 22, no. 1, pp. 62-67, 2006.

[34] T. Horbach, C. Götz, T. Kietzmann, and E. Y. Dimova, "Protein kinases as switches for the function of upstream stimulatory factors: implications for tissue injury and cancer," Frontiers in Pharmacology, vol. 6, 2015.

[35] M. Sirito, Q. Lin, J. M. Deng, R. R. Behringer, and M. Sawadogo, "Overlapping roles and asymmetrical crossregulation of the USF proteins in mice," Proceedings of the National Academy of Sciences, vol. 95, no. 7, pp. 3758-3763, 1998.

[36] V. S. Vallet, M. Casado, A. A. Henrion et al., "Differential roles of upstream stimulatory factors 1 and 2 in the transcriptional response of liver genes to glucose," Journal of Biological Chemistry, vol. 273, no. 32, pp. 20175-20179, 1998.

[37] K. Tatemoto, M. Carlquist, and V. Mutt, "Neuropeptide Y-a novel brain peptide with structural similarities to peptide YY and pancreatic polypeptide," Nature, vol. 296, no. 5858, pp. 659-660, 1982.

[38] E. Carstens, T. Follansbee, and M. Carstens, "The challenge of basic itch research," Acta Dermato Venereologica, vol. 100, no. 2, pp. 3-9, 2020.

[39] M. F. Coronel, M. J. Villar, P. R. Brumovsky, and S. L. González, "Spinal neuropeptide expression and neuropathic behavior in the acute and chronic phases after spinal cord injury: effects of progesterone administration," Peptides, vol. 88, pp. 189195, 2017.

[40] K.-M. Schebesch, A. Brawanski, S. Bele et al., "Neuropeptide Y - an early biomarker for cerebral vasospasm after aneurysmal subarachnoid hemorrhage," Neurological Research, vol. 35, no. 10, pp. 1038-1043, 2013. 